\title{
Systematic evaluation of genome-wide metabolic landscapes in lactic acid bacteria reveals diet- induced and strain-specific probiotic idiosyncrasies
}

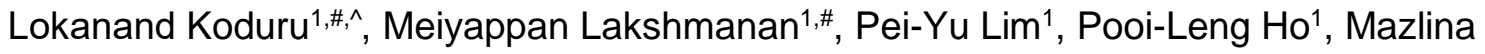 \\ Banu ${ }^{1}$, Doo-Sang Park ${ }^{2}$, Dave Siak-Wei Ow ${ }^{1, *}$ and Dong-Yup Lee ${ }^{1,3, *}$
}

${ }^{1}$ Bioprocessing Technology Institute, Agency for Science, Technology and Research

(A*STAR), 20 Biopolis Way, Singapore 138668, Singapore

${ }^{2}$ Korean Collection for Type Cultures (KCTC), Korea Research Institute of Bioscience and

Biotechnology (KRIBB), 181 Ipsin-gil, Jeongeup-si, Jeollabuk-do 56212, Republic of Korea

${ }^{3}$ School of Chemical Engineering, Sungkyunkwan University, 2066, Seobu-ro, Jangan-gu,

Suwon-si, Gyeonggi-do 16419, Republic of Korea

\#These authors contributed equally to this work

${ }^{*}$ Corresponding Authors

${ }^{\wedge}$ Current address: Institute of Molecular and Cell Biology, Agency for Science, Technology and Research (A*STAR), 61 Biopolis Drive, Singapore 138673, Singapore

\section{Corresponding authors details:}

Dave Siak-Wei Ow

Bioprocessing Technology Institute, Agency for Science, Technology and Research (A*STAR), 20 Biopolis Way, Singapore 138668; e-mail: dave ow@bti.a-star.edu.sg

and

Dong-Yup Lee

School of Chemical Engineering, Sungkyunkwan University, 2066, Seobu-ro, Jangan-gu, Suwon-si, Gyeonggi-do 16419, Republic of Korea; e-mail: dongyuplee@skku.edu 


\section{Abstract}

Lactic acid bacteria (LAB) naturally occur in animal and plant niches and are well-known to elicit several health benefits in humans. Yet, how they adapt their functional metabolic landscapes to diverse nutrient environments and synthesize relevant bioactive compounds remain unexplored across genera, species and strains. Hence, presented herein is a systematic framework for comprehensively characterizing the genome-wide metabolisms of six representative LAB by combining multi-omics data with in silico modeling. We analyse the differences in their growth and cellular fitness, biosynthetic capability of health-relevant compounds, i.e., postbiotics, and probable interactions with 15 common gut microbiota under 11 virtual dietary regimes, and show such attributes are diet- and species-specific. Particularly, some LAB exhibit a desirable balance between synthesis of beneficial postbiotic compounds, positive interactions with beneficial gut commensals, and the ability to colonize and persist in gut environment. We also observe that "high fat-low carb" diets likely lead to detrimental outcomes in most LAB. Our results clearly highlight that probiotics are not "one size fits all" commodities and need to be formulated in a personalised manner for their use as dietary supplements and live biotherapeutics. Overall, the proposed framework will systematize the probiotic administration and could also widen the strain repertoire.

Keywords: Lactic acid bacteria, smart probiotics, systems biology, diets, microbial interactions 
bioRxiv preprint doi: https:/doi.org/10.1101/2021.06.20.449192; this version posted June 21, 2021. The copyright holder for this preprint (which was not certified by peer review) is the author/funder, who has granted bioRxiv a license to display the preprint in perpetuity. It is made available under aCC-BY-NC-ND 4.0 International license.

'Lactic acid bacteria (LAB)' refers to a group of microaerophilic, Gram-positive bacteria which primarily ferment the hexose sugars into lactate ${ }^{1}$. LAB include several genera, Enterococcus, Lactobacillus, Lactococcus, Leuconostoc, Oenococcus, Pediococcus and Streptococcus, with functional classification into three major fermentative groups: homolactic, facultative heterolactic and obligative heterolactic, depending on the fermentation products which result from carbohydrate catabolism². LAB are indigenous species in human food habitat and are ubiquitously found in various nutrient-rich niches such as milk environments, vegetables and meats ${ }^{3}$. Moreover, they also constitute a part of the human microbiome in the large and small intestines and colon mucosal layers, exhibiting complex molecular cross-talk with the host and other microbiota to confer several beneficial health effects, e.g., antibacterial ${ }^{4-7}$, immunomodulatory and cytokine stimulatory ${ }^{8,9}$, free radical scavenging ${ }^{10,11}$ and antitumor activities $^{12,13}$. LAB have thus been a popular choice to maintain a good gut homeostasis and/or to ameliorate microbiota dysbiosis as it has been comprehensively reviewed elsewhere ${ }^{14,15}$.

Although certain LAB such as Lactobacillus plantarum, Lactobacillus salivarius and Lactobacillus casei are generally used as probiotics, their beneficial effects could be highly variable across different species, genera and strains. While several studies have extensively demonstrated the variations via both in vitro and in vivo experiments ${ }^{16-23}$, some have also reported a few commensurate health-promoting effects across various strains or even species $^{24-27}$. Such conflicting observations possibly stem from a myriad of factors including the differences in evaluation methodology and the distinct mechanisms of action: while some beneficial effects, e.g. bile resistance ${ }^{28}$ or mucosal adhesion ${ }^{29,30}$, could be common across even genera ${ }^{31}$, others might be niche- or strain-specific, e.g. synthesis of bioactive compounds, i.e. postbiotics. Therefore, it is of high importance to comprehensively examine the unique metabolic capabilities of $L A B$ and uncover their nutritional preferences and biosynthetic functions of various postbiotics in a context-specific manner. In this regard, the current availability of complete genome sequences for more than 1000 LAB strains ${ }^{32,33}$ has facilitated only a handful of comparative genomic analyses hinting at the plausible diversity in nutrient 
uptake and the ability to produce various antimicrobial substances ${ }^{34,35}$. Here, for the first time, we systematically evaluate the genome-wide functional metabolic capabilities of six representative $L A B$ from different genera, species and fermentative groups, through an integrative framework based on comparative genomics, transcriptomics and in silico modelling, together with their growth and biochemical profiles obtained from a newly formulated chemically defined medium (CDM). Such comparative analyses allow us to elucidate the probiotic determinants and probable interactions of LAB with common gut commensal and pathogenic microbes under various dietary regimes.

\section{Results}

\section{A common chemically defined medium for the unbiased evaluation of LAB growth characteristics}

We selected six representative strains, L. plantarum WCFS1 (LbPt), L. casei subsp. casei ATCC 393 (LbCs), Lactobacillus salivarius ATCC 11741 (LbSv), Lactobacillus fermentum ATCC 14931 (LbFm), Lactococcus lactis subsp. cremoris NZ9000 (LcLt) and Leuconostoc mesenteroides subsp. mesenteroides ATCC 8293 (LeMt), from three different genera in order to cover a wide range of LAB across different species as well as diverse functional groups. Note that although many Lactobacillus species are well established as probiotics, here, we purposely chose representatives from other well-studied genera of LAB, Leuconostoc and Lactococcus, to provide a good balance in terms of species diversity. Following the selection of strains, we newly formulated a chemically defined medium which supports the growth of all LAB tested. Design of CDM is highly necessary to enable an unbiased and comprehensive metabolic characterisation of all six LAB strains which are generally auxotrophic to several nutrients, particularly amino acids and vitamins. Here, it should be emphasized that although rich nutrient media such as M17 or Lactobacillus broth might support their growth, the chemically undefined components in the media may introduce batch-batch variations and uncertainties to substrate/product measurements. We initially cultivated all six LAB in a CDM which was originally developed to grow Lactococcus and Streptococcus species ${ }^{36}$. However, 
this medium was only able to support the growth of LcLt and LbSv. Therefore, we modified it iteratively by including several key metabolites based on the known growth requirements of each $L A B$, hereafter referred as $L A B$ defined medium (LABDM), for their unrestricted growth. Importantly, we eliminated the need for preparing multiple micronutrient component stocks of vitamins and minor salts in LABDM by replacing them with yeast nitrogen base (YNB), which is a defined commercial formulation of micronutrients widely used for yeast cultivation. The optimal supplementation of YNB ensured proper growth with minimum possible lag phase for most LAB strains (see Methods and Supplementary Dataset S1).

We next cultivated the LAB strains in LABDM and evaluated their batch growth characteristics under anaerobic conditions. Of them, LbPt and LbSv grew much faster, i.e. smaller doubling time than others (Figure 1a; p-val $=0.0077$, Kruskal-Wallis test). However, LbSv exhibited significantly longer lag phase compared to other LAB (Figure 1b and Supplementary Figure S1; -val $=0.0083$, Kruskal-Wallis test). The concentrations of major nutrients such as glucose and amino acids, and primary by-product, lactate, in the LABDM were also monitored over the exponential phase. No significant difference was observed in the biomass yield from glucose across LAB although obligate heterofermentative LAB (LeMt and LbFm) showed slightly lower yields (Figure 1c; p-val $=0.0186$, Kruskal-Wallis test), indicating their limited ability to catabolize amino acids and other nutrients for energy production, concordant with our previous study ${ }^{37}$.

Comparative genomic analysis of LAB reveals their pan genomic basis of probiotic capabilities

To understand how the probiotic characteristics vary across LAB, we conducted a thorough comparative genomic survey. We first constructed a phylogenetic tree using the genome sequences of all six species considered here with four other $L A B$ and an outgroup member, Bacillus subtilis (BcSt) (see Methods). While most Lactobacillus strains cluster together, interestingly, LeMt and BcSt are also placed in the same cluster (Figure 1d). This confirms a previous observation that Lactobacillus genus is paraphyletic ${ }^{34}$ and underlines the remarkable 
genetic diversity of LAB species, which could be a result of their adaptation to various nutrientrich niches. Furthermore, LAB genomes vary widely in size $(2.55 \pm 0.84 \mathrm{Mb})$, where LbPt and LbFm have the largest $(3.84 \mathrm{Mb})$ and smallest $(1.86 \mathrm{Mb})$ genomes, respectively (Supplementary Table S1), signifying the potential variations in probiotic traits among them. Such large variations in the genome size could possibly be a result of significant horizontal gene loss/gain in the evolutionary process, further confirming the ongoing process of genome degeneration as indicated by the presence of pseudogenes in most of the $L A B^{34}$.

We next quantified the genetic redundancy across $L A B$ on the basis of orthologous genes to comprehensively characterize how their genomic catalogues differ from each other (see Methods). A total of 627 unique "core" genes to be present commonly and 1567 "pan" genes that are either unique to a particular $L A B$ or shared in a few were identified, thus emphasizing the extraordinary genetic diversity in LAB despite their small genome sizes (Figure 1e). Functional annotation of the core and pan genome (see Methods) highlighted that housekeeping processes such as translation, ribosomal structure and biogenesis, replication (17\% compared to $1 \%$ ) and post-translational modification, protein turnover, chaperones (5\% compared to $2 \%$ ) are highly enriched in the core genome compared to the pan genome. In addition, a few metabolic pathways such as lipid and nucleotide metabolisms, were highly conserved across LAB species (Figure 1f). On the other hand, more enriched in pan genome include carbohydrate, amino acid, inorganic ion, coenzyme and other secondary metabolism, and some non-metabolic categories such as transcription and function unknown. Significant enrichment of metabolic genes in pan genome, particularly carbohydrate and amino acid metabolisms, pinpoints the differences in their preferential adaptation to nutrient environments such as milk, plant and animals ${ }^{38-40}$ as well as the variations in their probiotic capabilities $^{41}$. Moreover, the high proportion of genes with unknown function (980/3245) in LAB pan genome hints at the gaps in the current understanding and the need for a comprehensive functional annotation of such genes. LAB also synthesize a variety of proteins for resisting acid, oxidative and bile stresses, conferring anti-microbial activities, protein lysing 
bioRxiv preprint doi: https:/doi.org/10.1101/2021.06.20.449192; this version posted June 21, 2021. The copyright holder for this preprint (which was not certified by peer review) is the author/funder, who has granted bioRxiv a license to display the preprint in perpetuity. It is made available under aCC-BY-NC-ND 4.0 International license.

proteases and anchor cells on host mucosal layer surfaces, which constitute important probiotic features. We mined all six LAB genomes and observed that LAB with larger genomes, i.e. LbPt and LbCs, synthesize multiple cell envelope proteinases (CEPs) and peptidases (Figure 1g; see Methods and Supplementary Results). Taken together, these findings suggest the dominant role of pan genomic features in determining the probiotic capabilities in LAB.

\section{Genome-wide transcriptome sequencing of $L A B$ reveals unique gene expression profiles irrespective of their taxonomic groups}

In order to delve deeper insights into the genome-wide transcriptional regulation among various taxonomic groups, we collected RNA at the mid-exponential phase from all strains and performed RNA-seq. At the outset, we compared the global gene expression profiles by analysing the distribution of genes which fall under various expression ranges. LbSv and LbFm have large number of genes in low expression range while LbPt, LbCs and LeMt have gene expression distribution slightly skewed towards high expression range (Figure $\mathbf{2 a}$ and Supplementary Figure S2). Subsequent enrichment analysis of NOG terms in these gene expression categories (see Methods) identified housekeeping functions, i.e. translation, ribosomal structure and biogenesis (FDR adj. p-val $=8.830 \times 10^{-30}-1.805 \times 10^{-17}$, Fisher's exact test), in high expression range (Figure $\mathbf{2 b}$ ). We also noted that carbohydrate transport and metabolism is significantly enriched (FDR adj. $p$-val $=1.341 \times 10^{-10}-3.128 \times 10^{-7}$, Fisher's exact test) in low expression range particularly for $\mathrm{LbPt}$, LbCs and LcLt, indicating that the metabolism of LAB with larger genomes is further regulated at the transcriptional level. Noticeably, we also found a wide spread expression of genes with unknown function, highlighting the need to further characterize their role in governing LAB phenotype.

In addition to the global transcriptome analysis, we compared the interspecies transcriptomic landscapes and their predisposition to various metabolic and probiotic capabilities by first identifying the 549 one-one orthologues (including 195 metabolic genes), and examining their normalized expression values using a set of housekeeping genes to 
bioRxiv preprint doi: https:/doi.org/10.1101/2021.06.20.449192; this version posted June 21, 2021. The copyright holder for this preprint (which was not certified by peer review) is the author/funder, who has granted bioRxiv a license to display the preprint in perpetuity. It is made available under aCC-BY-NC-ND 4.0 International license.

correct for the species-specific bias (see Methods and Supplementary Figure S3). The expression profiles of these genes were analysed using two individual and complementary metrics: i) principal component analysis (PCA) and ii) correlation in a pairwise manner. Surprisingly, the gene expression patterns are markedly different between phylogenetically closer species such as LbSv and LbCs (Spearman correlation, $r=0.673$ ) while it is highly similar in distant species (Figures 2c-d), e.g. LbFm and LcLt (Spearman correlation, $r=0.96$ ). Hierarchical clustering of differentially expressed orthologous genes resulted in 9 major groups where each cluster is mainly represented by genes that are upregulated in a particular LAB (Figure 2e). Enrichment of functional categories in these genes further revealed that the carbon metabolism and several growth-related biological processes such as DNA replication (FDR adj. p-val $=3.162 \times 10^{-17}$, modified Fisher's exact test), ribosome and DNA mismatch repair (FDR adj. p-val $=0.0031$, modified Fisher's exact test) were upregulated in LAB with larger genomes such as LbPt, LbCs and LcLt, thus providing further evidences to why these species grow much faster than others (Figure 2f and Supplementary Figure S4). On the other hand, LeMt show high gene expression of anaerobic respiration (FDR adj. $p$-val $=0.0034$, Fisher's exact test), i.e. F1ATPase and menaquinone biosynthesis, indicating that redoxcontrolled metabolism governs the fastidious growth as reported earlier ${ }^{37}$. We also observed a high expression of certain postbiotics biosynthetic pathways in $\mathrm{LbCs}$ and $\mathrm{LbPt}$, while it is lower in LbFm and LbSv.

Since the transcriptional regulation of each LAB is significantly different even among the phylogenetically closer species, we further performed a PCA focusing on metabolic gene expression. The first and second PCs account for $\sim 32 \%$ and $25 \%$ variations, respectively, with major contributions from the functional categories relevant to growth (alternate carbon metabolism, aminoacyl-tRNA biosynthesis, heterolactic fermentation, oxidative phosphorylation and starch and sucrose metabolism) and biosynthesis of bioactive compounds (protonate metabolism, butanoate metabolism, lipoteichoic acid biosynthesis and vitamin metabolism) (Figure $\mathbf{2 g}$-i). These results clearly suggest that gene expression 
bioRxiv preprint doi: https:/doi.org/10.1101/2021.06.20.449192; this version posted June 21, 2021. The copyright holder for this preprint (which was not certified by peer review) is the author/funder, who has granted bioRxiv a license to display the preprint in perpetuity. It is made available under aCC-BY-NC-ND 4.0 International license.

variations among $L A B$ can be better captured by their ability to adapt to various environments and produce relevant bioactive compounds rather than their taxonomic differences.

\section{Genome-scale modeling delineates the diverse metabolic repertoire and the predominant use of low energy-yielding pathways in LAB strains}

As the comparative genomics and transcriptomics analyses revealed an unprecedented divergence in metabolic and physiological capabilities of LAB, we reconstructed new genomescale metabolic models (GEMs) for LbCs, LbFm and LbSv and updated the existing ones for $\mathrm{LbPt}^{42}$, LcLt ${ }^{43}$ and LeMt ${ }^{37}$, following the standard procedure ${ }^{44}$ (see Methods) to further probe their functional metabolic states. The resulting GEMs contained $686 \pm 139$ genes, $1042 \pm 65$ reactions and $906 \pm 29$ unique metabolites on an average, where LbFm and LbPt represented the smallest and largest models, respectively (Supplementary Table S2). We subsequently calculated the metabolic distances between pairs of any two GEMs using their genome-wide reactome and observed that all Lactobacillus strains shared more reactions than taxonomically distant LeMt and LcLt (Supplementary Figure S5). We then compared the LAB GEMs to identify the "core" and "pan" metabolic repertoire, as such finding $49 \%$ of the total reactions conserved (Figure 3a). The highly conserved metabolic subsystems are aminoacyltRNA biosynthesis (100\%), lysine biosynthesis (84\%), peptidoglycan biosynthesis (78\%), glycerophospholipid metabolism (72\%) and fatty acid biosynthesis (68\%). The pan reactome, on the other hand, was largely represented by carbohydrate and amino acid metabolism, confirming the high diversity of LAB in their ability to catabolize various nutrients as highlighted by comparative genomic analysis. We also investigated the variations in the amino acid auxotrophy (Figure $\mathbf{3 b}$ ) and noticed the absence of several reactions in the relevant biosynthetic pathways (Figure 3c).

Following the reconstruction of GEMs, we validated the model predictions using the batch culture data of LAB grown in LABDM (see Methods). The initial in silico growth rates predicted by flux balance analysis (FBA) were comparable to experimentally measured ones. However, most LAB models failed to simulate the lactate secretion, releasing other 
fermentative products instead. This motivated us to incorporate additional kinetic constraints for accurately predicting the LAB phenotype. In this regard, implementation of FBA with additional macromolecular crowding constraints (FBAwMC) has been reported to successfully describe the low-yielding acetate overflow in Escherichia colit ${ }^{45}$, the Warburg effect in cancer cells $\mathrm{s}^{46,47}$ and the role of redox cofactors in the switch between low and high yield metabolisms in Saccharomyces cerevisiae and $\mathrm{LcLt}^{48}$. Therefore, we subsequently formulated an approach based on FBAwMC employing experimentally measured crowding coefficients to investigate the functional metabolic states of each LAB (see Methods, Supplementary Methods, Supplementary Tables S3-S4 and Supplementary Figures S6-S7). Remarkably, the growth rates and lactate secretion rates predicted were highly consistent with the culture experiments (Figures $\mathbf{3} \mathbf{d}$ and $\mathbf{3 e}$ ), thus confirming that the predominant use of low energy yielding pathways is a hallmark of $\mathrm{LAB}^{42,49}$.

In silico analysis of LAB GEMs highlights the diet-specific patterns in probiotic colonization and postbiotic synthesis

Previously, several cohort-based studies have reported that the gut microbiome composition is more sensitive to the dietary habits than the ethnic or geographical makeup ${ }^{50-52}$. Similarly, we hypothesize that some probiotics may potentially survive and produce beneficial compounds much better in certain diets, which could be tested in silico. We explored the probiotic capability of LAB under 11 different diet conditions including vegetarian, vegan, EU average, Mediterranean, $\mathrm{DACH}$, high protein, gluten free, high fibre, diabetes, high fat-low carb, and high fat-high carb by resorting to the model-driven analysis (see Methods). The resulting growth patterns signified very distinct diet-specific phenotypes: most of the LAB, especially obligate homofermentative, preferred diabetes and high fibre diets whereas all of them grew very slowly in high fat diets (Figures 4a-4f and Supplementary Figure S8; p-val $<1 \times 10^{-150}$, Kruskal-Wallis test). The high fibre and diabetes diets are usually rich in inulin type fructooligosaccharides which are catabolized via high ATP yielding pathway involving the (phospho-)fructo-furanosidase (BfrA or $S a c A / S c r B$ ) enzyme, and thus giving rise to the increased growth rates ${ }^{53}$. LAB do not grow well in high fat-low carb diets since they cannot 
bioRxiv preprint doi: https:/doi.org/10.1101/2021.06.20.449192; this version posted June 21, 2021. The copyright holder for this preprint (which was not certified by peer review) is the author/funder, who has granted bioRxiv a license to display the preprint in perpetuity. It is made available under aCC-BY-NC-ND 4.0 International license.

utilize fatty acids as primary carbon substrates for energy generation although fatty acids are primary constituents for microbial cell walls ${ }^{54}$. Interestingly, we also observed minimal variations in growth rates of heterofermentative LAB (LbFm and LeMt) across different diets, mainly due to the similar levels of ATP produced from diverse carbohydrates via phosphoketolase pathway and their limited capacity to use amino acids as alternative energy sources $^{37}$.

We subsequently evaluated LAB potentials to produce relevant bioactive compounds (postbiotics) that are beneficial to health, such as the short-chain fatty acids (SCFAs) including butyrate, propionate and acetate. To do so, we computed the theoretical maximum yield of various postbiotic compounds while constraining the biomass flux at $50 \%$ of its maximum achievable value (see Methods for details). It should be noted that some metabolites secreted by LAB are also known to aggravate health conditions. For example, lipoteichoic acids (LTA) promotes pro-inflammatory responses such as TNF- $\alpha$ induction ${ }^{61}$. Thus, their overall benefit to the host depends on the balance between the production of beneficial and detrimental metabolites. LbPt and LcLt have high amount of growth-coupled production of various beneficial postbiotic compounds such as SCFAs, amino acids and vitamins across all diets comparatively (Figure $\mathbf{4 h}$ ). LbCs and LbSv also secrete similar levels of SCFAs across various diets although they are incapable of synthesizing vitamins and amino acids as high as LbPt and LcLt. On the other hand, LeMt and LbFm synthesized relatively low levels of SCFAs. Similar to the growth patterns, the production level of beneficial metabolites is higher in carbohydrates rich diets, e.g. EU average, $\mathrm{DACH}$, high fibre and diabetes, while it is low in high fat diet. Interestingly, plant-based diets such as vegan, Mediterranean and diabetes consistently enabled several LAB to produce higher amounts of vitamins. Since unique pattern of the gene expression in fermentation pathways was observed for each $L A B$, we again predicted their postbiotic production with additional constraints based on transcriptome data. These simulations show that while the production of primary metabolites such as lactate and acetate is not significantly affected by gene expression, other compounds are partially 
regulated at transcript level in few species including LbCs and LeMt (Supplementary Figure S9). Here, it should be noted that while we analyzed the biosynthetic capability of LAB, some of them could also consume/degrade the postbiotics including certain amino acids and vitamins synthesized by other LAB as suggested by the auxotrophic analysis (Figure $3 \mathbf{b}$ ), and may partly compromise the beneficial effects.

\section{Pairwise metabolic interactions of LAB unravel their overall positive associations with commensal species}

Probiotics have also shown to selectively interact with the gut flora and alter their abundance ${ }^{62,63}$, particularly in the context of their use as live biotherapeutics. Here, we investigated the pairwise metabolic interactions of each LAB strain with representative probiotics (Bifidobacterium adolescentis and other 5 LAB), commensals (Akkermansia muciniphila, Bacteroides thetaiotaomicron, Enterococcus faecalis, Escherichia coli W3110, Faecalibacterium prausnitzii), opportunistic pathogens (Pseudomonas putida, H. pylori, Klebsiella pneumoniae, Pseudomonas aeruginosa, Escherichia coli O157:H7) and pathogens (Salmonella enterica ser. typhimurium, Shigella flexneri, Shigella sonnei, Staphylococcus aureus) under the 11 diets (see Methods). LbCs, LbFm, LbSv and LcLt enhance the growth of commensals while they were either neutral or detrimental to pathogens (Figure 5, Supplementary Figures S10-S15). LbCs and LbFm particularly cross-feed F. prausnitzii and A. muciniphilia, which are widely recognized for their protective role in many metabolic and inflammatory disorders. However, LbPt and LeMt displayed mixed results with no clear distinction between beneficial and detrimental species; they promoted both the growth of commensals such as $F$. prausnitzii and $A$. muciniphilia and pathogenic bacteria such as Salmonella and Shigella. In addition to strong organism-specific patterns in microbial interactions, we also observed subtle diet-specific patterns. Carbohydrate and protein-rich diets such as Mediterranean, $\mathrm{DACH}$, high protein and high fibre, promoted the growth of commensal and probiotic species in LbCs, LbPt and LeMt (Figure 5). 
We conducted an extensive literature survey to further validate the simulation results, which showed that LAB could have positive interactions with certain commensal strains. For example, a previous study ${ }^{64}$ reported that the culture supernatants from LcLt and Lactobacillus paracasei stimulated $F$. prausnitzii growth by supplementing B-vitamins and lactate/acetate. However, we could not find any evidences of similar interactions between LAB and another keystone gut commensal, A. muciniphilia. Therefore, we newly performed cell culture experiments to evaluate such cross-feeding between $\mathrm{LAB}$ and $A$. muciniphilia, $B$. thetaiotamicron and E. coli (see Methods, Supplementary Results, Supplementary Figure S16 and Supplementary Dataset S2). Among the total 18 combinations experimentally evaluated (6 LAB $\times 3$ commensal strains), 16 of them agreed well with model predictions (F1score $=0.8333$ ). These results demonstrate the high consistency of model predictions and the utility of GEMs in evaluating microbial metabolite cross-feeding.

\section{Discussion}

While conventional probiotics have been generally developed as food supplements to maintain a good gastrointestinal health, next generation probiotics are expected to provide preventive and therapeutic means to intervene with various diseased states ${ }^{65}$. In this regard, a key challenge is to design combinatorial probiotic formulae based on their synergistic interactions within and with the microbiome. Strain-specific differences and influence of environmental factors including diet could further confound their application ${ }^{55}$. Moreover, there exists no rational framework for screening and functionally classifying probiotics targeting human health, although the Food and Agriculture Organization (FAO) of the United Nations (UN) has currently placed certain guidelines which highly rely on various experimental methods, such as those involving animal models ${ }^{66}$. However, it is impractical to test the efficacy of all candidates and their combinations at the preclinical stage. Therefore, herein we provide a first-pass rational screening procedure to earmark promising candidates using an in silico model-guided approach which could be further verified in vivo. 
bioRxiv preprint doi: https:/doi.org/10.1101/2021.06.20.449192; this version posted June 21, 2021. The copyright holder for this preprint

(which was not certified by peer review) is the author/funder, who has granted bioRxiv a license to display the preprint in perpetuity. It is made available under aCC-BY-NC-ND 4.0 International license.

Our comparative genomic and transcriptomic analyses suggested LbPt and LbCs as the common LAB with a higher proportion of functionally relevant genes supporting gastrointestinal survival via the resistance to acid, bile and oxidative stress, production of bacteriocins and CEPs, and mucosal adhesion. Interestingly, both LAB have relatively larger genomes and coincidentally belong to the facultative heterofermentative group. Another interesting finding is the extraordinary diversity of LAB pan genome, especially enriched on carbohydrate and amino acid metabolism, which motivated us to explore the functional metabolic capabilities as well as the cellular fitness across different diet conditions. Indeed, our model-driven analysis confirmed diet-specific patterns where high fat-low carb (ketogenic) diets lead to poor probiotic performance and diets rich in inulin type fructooligosaccharides is preferable, in general. Consistent with our results, recent clinical studies have also shown that diets rich in high fat-low carb cause unfavourable changes in gut microbiome and reduction in fecal SCFAs which could be due to the elimination of probiotic bacteria ${ }^{67-69}$. These observations clearly demonstrate the need to either select appropriate probiotics depending on the dietary habit of a person or administer them as synbiotics, i.e., probiotics with relevant 'prebiotics', to enable their efficient functioning in gut. Such rational administration of probiotics becomes even more significant for live biotherapeutics. In this regard, although our results indicate LbPt, LcLt and LeMt to have highest capabilities for the postbiotic production, they also synthesize increased levels of LTA which is highly implicated in the stimulation of proinflammatory cytokines ${ }^{61}$, and thus caution must be exercised while recommending them to patients with inflammatory bowel disease (IBD). Our simulations also hinted that LbCs and LbFm could be promising probiotic candidates to treat/manage IBD as they both selectively promoted the growth of two commensals (A. muciniphilia and F. prausnitzii) that are reported to have a strong inverse correlation with $\mathrm{IBD}^{70}$. Numerous earlier studies have reported that LbCs strains consistently confer anti-inflammatory and protective effects against IBD and colorectal cancer ${ }^{71-76}$. On the other hand, LbPt and LeMt displayed positive interactions with both pathogens and commensals growth, and thus may preferably be avoided for treating immunocompromised patients whose natural ability to eliminate gastrointestinal pathogens is 
generally limited $^{77}$. These observations demonstrate the advantage of adopting a multi-omics based systems approach compared to the widely used comparative genomics analysis.

In summary, the current study highlights that the fitness and postbiotic synthesis capabilities of probiotics are diet-dependent while their interactions with gut microbes are largely organism-specific. Our in silico simulation results clearly indicate that while high fiber diets promote the probiotic growth and functionality, high fat diets lead to poor performance, which is in high concordance with previously published clinical data ${ }^{67-69}$. However, we urge caution in extrapolating inferences from the presented framework as the annotation of microbial genomes vary considerably. In addition, some of the probiotic evaluation aspects, e.g. colonization, are still uncertain ${ }^{55}$, which can be simply addressed by further revising the presented in silico analysis. Despite such restraints, the systematic framework proposed here plugs the gap between comparative genomic analyses and in vivo evaluation. It also seeds the foundation for model-guided selection of relevant probiotics, and can be further extended by accounting relevant confounding factors in clinical settings, such as host-genetics and actual diets, through community-wide efforts to foresee well-controlled clinical trials. Overall, the proposed approach is poised to accelerate the characterization of probiotic capabilities of multiple candidates beyond the conventional Lactobacillus and Bifidobacterium species, thus speeding up the rational design of smart probiotics in near future.

\section{Methods}

\section{LAB strains, media and growth conditions}

LAB strains, Leuconostoc mesenteroides subsp. mesenteroides ATCC 8293, Lactobacillus casei subsp. casei ATCC 393, Lactobacillus plantarum WCSF1, Lactobacillus salivarius ATCC 11741, Lactobacillus fermentum ATCC 14931 were from ATCC, and Lactococcus lactis subsp. cremoris NZ9000 was from Boca Scientific. The lyophilized cultures were first revived by aseptically transferring the contents of the ampoule into $10 \mathrm{ml}$ each of MRS and GM17 media, equally. The revived cultures were then sub-cultured using the same media to ensure 
bioRxiv preprint doi: https:/doi.org/10.1101/2021.06.20.449192; this version posted June 21, 2021. The copyright holder for this preprint (which was not certified by peer review) is the author/funder, who has granted bioRxiv a license to display the preprint in perpetuity. It is made available under aCC-BY-NC-ND 4.0 International license.

stability. A chemically defined medium capable of supporting all six LAB strains (LABDM) was developed by iteratively modifying components of a previously established $\mathrm{CDM}^{36}$ (see Supplementary Dataset S1). Seed LAB cultures for fermentation experiments were prepared by transferring the $10 \%(\mathrm{v} / \mathrm{v})$ cultures to $\mathrm{LABDM}$ and incubating them overnight at $30^{\circ} \mathrm{C}$. Biological triplicates of the $L A B$ were grown in $50 \mathrm{~mL}$ conical tissue culture tubes (Thermo Fisher Scientific) as static cultures with added reducing agent, sodium thioglycolate (1g/L), and no head-space to maintain anaerobic conditions. A separate culture tube was maintained for each time point, to avoid aeration during sampling.

\section{Growth and biochemical analysis of culture supernatants}

Cells and supernatants were harvested from the biological triplicate LAB cultures cultivated in LABDM at regular time intervals spanning the exponential growth phase for various growth and other biochemical assays. Optical density at 600nm (OD600) was measured using Shimadzu UV-1700 spectrophotometer. Dry cell weights (DCW) were obtained by centrifuging known volume of cell cultures, subsequently drying the pellet at $100^{\circ} \mathrm{C}$ overnight, and weighing them using a balance. Standard curves of OD600 vs gDCW were plotted to estimate the conversion factors. Glucose and lactate profiles at different time intervals spanning exponential phase were analyzed using YSI® biochemistry analyzer. Amino acid profiles were measured using Waters ACQUITY-UPLC system, AccQ•Tag ${ }^{\text {TM }}$ Ultra Column $(2.1$ x 100 mm) and AccQ-Tag derivatization kit, following the manufacturer instructions. Note that biological triplicates were employed for all sample measurements.

\section{Cell volume and cell number measurements}

LAB single cell volumes were estimated by approximating the shapes of LcLt and LeMt to spherical, and LbPt, LbCs, LbSv and LbFm to cylindrical dimensions. Average cell diameters and lengths of at least a hundred cells measured using an optical microscope at $100 \mathrm{X}$ magnification under oil immersion were used to estimate single cell volumes. Bacterial cultures were appropriately diluted before microscopic measurements to ensure proper dispersal of 
cells. Cell numbers in a given volume of the same diluted culture were enumerated using plating and automatic CFU counter (Scan 1200, Interscience, Saint Nom, France).

\section{Determination of total protein content}

Total protein content was measured using Bradford assay ${ }^{81}$. Briefly, cells pellet obtained from culture of known volume and OD600 were suspended in $1 \mathrm{~mL}$ ice cold lysis buffer containing $10 \mathrm{mM}$ Tris-HCL, pH 8.0, $1 \mathrm{mM}$ EDTA, and $100 \mathrm{ul}$ of lysozyme $(10 \mathrm{mg} / \mathrm{mL})$, and incubated on ice for $15 \mathrm{~min}$. Next, the cells were sonicated at a frequency of $20 \mathrm{kHz}$ for $10 \times 30 \mathrm{sec}$ with 1 min interval between each sonication cycle. Ten rounds of sonication resulted in "close to complete" protein release, after which the protein concentration saturated. Bradford protein assay kit (Bio-Rad®) with standard Bovine Serum Albumin (BSA) solution was used to estimate the protein concentration in the sonicated samples after sufficient dilution, which were then converted to total protein content per gram DCW using appropriate conversion and dilution factors.

\section{Total RNA extraction and purification}

To extract the total RNA, $10 \mathrm{~mL}$ aliquots were harvested from the biological triplicate $\mathrm{LAB}$ cultures cultivated in LABDM at time points that correspond to their respective mid-exponential phases. It was first mixed with $10 \mathrm{~mL}$ of Qiagen RNAprotect reagent and incubated for $10 \mathrm{~min}$ at ambient temperature to stabilize RNA. Bench and biosafety cabinet surfaces used for RNA work were decontaminated with RNaseZAP (Thermo Fisher Scientific) to minimize contact of samples with RNase. Half of the sample aliquotes $(10 \mathrm{~mL})$ containing RNAprotect reagent were centrifuged at $6000 \times \mathrm{g}$ for $10 \mathrm{~min}$. The remaining samples were stored at $-80^{\circ} \mathrm{C}$ up to 4 weeks for any later use. Prior to RNA extraction, the cell pellets were treated with $1 \mathrm{~mL}$ icecold lysis buffer containing $10 \mathrm{mM}$ Tris-HCL, pH 8.0, $1 \mathrm{mM}$ EDTA, and $100 \mathrm{uL}$ of lysozyme (10 mg/mL), all prepared using RNase-free water, and incubated for $30 \mathrm{~min}$. The lysed solution was further subjected to mechanical cell disruption involving nuclease-free glass beads and thermomixer to ensure maximum release of RNA. Qiagen RNeasy Mini kit was used to extract total RNA from the cell pellets following manufacturer instructions. DNase treatment was 
performed using Turbo DNA-free kit (Thermo Fisher Scientific) to remove any genomic DNA contamination. Integrity and quality of the extracted RNA was initially checked by the presence of $16 \mathrm{~s}$ and $23 \mathrm{~s}$ rRNA in samples subjected to agarose gel electrophoresis and later confirmed using Agilent Bioanalyzer. Bacterial Ribo-Zero Magnetic kit (Illumina) was used to deplete rRNA and enrich mRNA in the samples, following manufacturer's instructions. Agilent Bioanalyzer was used to ensure the depletion of rRNA from all samples before the preparation of cDNA libraries.

\section{Preparation of cDNA libraries and RNA-sequencing}

Fragmentation of rRNA depleted mRNA samples and subsequent preparation of cDNA libraries were performed using Illumina TruSeq Stranded mRNA Library Preparation kit (Low Sample Protocol), following manufacturer's instructions. First cDNA strand synthesis in the reverse transcription polymerase chain reaction (RT-PCR) was performed using the reagents supplied by the manufacturer along with the SuperScript II reverse transcriptase (Thermo Fisher Scientific). Indexed cDNA libraries were pooled and single-end sequenced on Illumina HiSeq 2500 Rapid V2 platform to 51 bp read length.

\section{Commensal culture with LAB supernatants}

Brain heart infusion $(\mathrm{BHI})$ broth supplemented with heme, $\mathrm{N}$-acetylglucosamine was used to culture all three commensal strains (A. muciniphila ATCC BAA-835, B. thetaiotaomicron VPI5482, and E. coli W3110). Media were purged with $\mathrm{N}_{2}$ gas prior to use, ensuring they are devoid of oxygen. $L A B$ strains were first statically grown in CDM overnight at $30^{\circ} \mathrm{C}$. Simultaneously, commensal bacteria were inoculated to $\mathrm{BHI}$ medium and allowed them to grow overnight at $37^{\circ} \mathrm{C}$. Supernatants from the LAB culture $10 \% \mathrm{v} / \mathrm{v}$ was then transferred to freshly prepared $\mathrm{BHI}$ in a culture tube. The overnight-grown commensal culture was seeded to this tube to a final OD600 of 0.1-0.2. All experiments were conducted in duplicates and were done in an anaerobic chamber. The culture tube was placed inside anaerobic jar and purged with $\mathrm{N}_{2}$ for 10 minutes before including the GazPak EZ sachets (Becton-Dickinson). After 
sealing, the jar was placed in a $37^{\circ} \mathrm{C}$ incubator. Growth of the commensal bacteria was recorded by measuring OD after 12 hours of incubation.

\section{Statistical analysis of $L A B$ growth characteristics}

LAB growth characteristics were evaluated on the basis of three parameters: maximum growth rate, time taken to reach mid-exponential phase and the biomass yield over glucose. Briefly, maximum growth rate is the maximum value of slope along the log transformed growth curve. Time to mid-exponential is the time required to reach half the maximum OD in the culture. Biomass yield over glucose is the ratio of LAB gDCW accumulated over the culture to that of g glucose consumed. Maximum growth rate and time to mid-exponential were calculated by fitting a logistic equation to the culture data using Growthcurver ${ }^{82}$.

\section{Comparative genomic analysis}

Reconstruction of phylogenetic tree: To understand the evolutionary perspective of $L A B$ strains examined in this study, phylogenetic tree was constructed from the whole genome sequences using Type (Strain) Genome Server (TYGS) ${ }^{83}$. Note that we also included four other LAB strains, Lactobacillus reuteri ATCC 53608, Lactobacillus delbrueckii subsp. bulgaricus ATCC 11842, Lactobacillus acidophilus NCFM, and Bacillus subtilis subsp. subtilis str. 168 , for this particular analysis. Whole genome phylogeny trees were reconstructed based on Genome BLAST Distance Phylogeny (GBDP) approach where all genome sequences were compared pairwise and intergenomic distances was calculated using the 'trimming' algorithm and distance formula $\mathrm{d} 5^{84}$. Phylogenetic trees with branch support were then inferred from intergenomic distances using $\mathrm{FastME}^{85}$ based on the original and pseudo-bootstrapped matrices, and visualized using Interactive Tree Of Life (iTOL) ${ }^{86}$.

Identification and functional annotation of LAB "core" and "pan" genome: In order to identify the "core" and "pan" genome, we first established the homology relationships between any pair of $L A B$ species using InParanoid ${ }^{87}$. Since InParanoid orthology detection is based on pairwise comparisons, we generated 15 InParanoid tables, identifying the orthologous genes for each genome pairs. Subsequently, we compared the list of one-to-one orthologs across 
the LAB using LbPt as reference, and established the "core" genes that are present in all species. The remaining non-unique gene count in each LAB is considered as "pan" genome. Functional enrichment of "core" and "pan" genome was performed using eggNOG mapper based on the NOG categories ${ }^{88}$. Note that NOG categories are similar to well-known COG categories but it is identified in an unsupervised manner.

Genomic comparisons for non-metabolic probiotic determinants: BLASTp orthology search was used to mine the LAB genomes for identifying ORFs encoding proteins for resisting acid, oxidative and bile stresses, protein lysing proteases and anchor cells on host mucosal layer surfaces. To do so, we first surveyed for genes known to be associated with probiotic characteristics in various LAB (See Supplementary Information). Appropriate cutoffs (Identity $>50 \%$, query coverage $>70 \%$, E-value $<1 \mathrm{E}-06)^{89}$ were used to select orthologs. Potential bacteriocin operons in the LAB genome was mined using BAGEL ${ }^{90}$.

\section{Transcriptome data analysis}

Transcriptome assembly and gene expression quantification: First, the quality of RNAseq reads were assessed with FastQC ${ }^{91}$. The adapters and low-quality reads were subsequently trimmed using Trimmomatic $\mathrm{v} 0.32^{92}$ and the trimmed reads were then aligned with the respective genome assemblies using STAR v 2.5.3a ${ }^{93}$. Finally, RSEM v1.3.0 $0^{94}$ was used to quantify expression levels in terms of counts, FPKM and TPM from the alignment files. Functional enrichment of gene expression categories: First, the genes are grouped into three groups based on TPM values: i) low expression range $(\log 2(T P M+1)<2.5)$, ii) medium expression range $(\log 2(\mathrm{TPM}+1)>2.5 \&<10)$ and iii) high expression range $(\log 2(\mathrm{TPM}+1)>$ 10). Subsequently, a p-value is calculated representing the significance of NOG category enrichment in different gene expression groups using the Fisher's exact test. The p-values was subsequently adjusted for false-discovery rates based on previously proposed method ${ }^{95}$. Comparison of gene expression in orthologous genes: The direct comparison of gene expression across LAB species in gene wise manner is not possible because their genome sizes vary as well as their genome functionalities are different. Therefore, in order to compare 
the gene expression levels of same gene functions across $L A B$, we first identified one-to-one orthologues as mentioned previously. We subsequently normalized the raw counts of these orthologues based on a set of housekeeping genes using RUVseq ${ }^{96}$. The housekeeping genes were identified using the following criteria: i) genes which have expression over 95 percentile range in each $L A B$ and ii) genes which are present in one-to-one orthologues list. Orthologous gene expression profiles were then compared using PCA and Spearman correlation in a pairwise manner.

Identification of differentially expressed genes: DEseq $2{ }^{97}$ was used to identify differentially expressed one-to-one orthologous genes across the 6 LAB. Note that we used the gene lengths in each LAB as an additional normalization factor in this step since the gene length will be different in each LAB for the same gene. Those genes with FDR adjusted p-values < 0.01 were classified as significantly differentially expressed genes.

Functional enrichment of differentially expressed genes: Differentially expressed genes were functionally enriched based on KEGG pathways and GO terms using Database for Visualization and Integrative Discovery (DAVID) ${ }^{98}$. Pathways and GO terms which have an FDR adjusted $p$-value $<0.01$ were only considered for further analysis.

\section{Reconstruction of LAB genome-scale models}

GEMs of LbSv, LbFm and LbCs were newly developed based on their respective genome annotations, whereas LbPt, LeMt and LcLt GEMs have been updated from the previously published ones ${ }^{37,42,43}$. The reconstruction/updating of GEMs involved standard procedures, which can be found elsewhere ${ }^{44}$.

To newly reconstruct GEMs, initially the metabolic pathway information based on genome annotations of the $L A B$, was collected from databases, including KEGG ${ }^{99}$ and MetaCyc ${ }^{100}$. The draft reaction networks were then assembled by combining information from these databases, along with the information based on gene orthologs of the existing $L A B$ models ${ }^{37,42,43}$. Next, an artificial reaction, known as biomass reaction, which is typically used as an objective function in constraint-based flux analysis to predict metabolic fluxes of 
bioRxiv preprint doi: https:/doi.org/10.1101/2021.06.20.449192; this version posted June 21, 2021. The copyright holder for this preprint (which was not certified by peer review) is the author/funder, who has granted bioRxiv a license to display the preprint in perpetuity. It is made available under aCC-BY-NC-ND 4.0 International license.

exponential growing cells ${ }^{101}$, was added to each newly developed model based on experimental and literature information (see Supplementary Dataset S3). Note that since protein constitutes the major macromolecular component of biomass, we measured the total protein content of different LAB experimentally and used it for biomass reaction formulations (Supplementary Dataset S3). Gaps in the these draft networks were then identified using gapFind algorithm ${ }^{102}$ and subsequently filled by adding new reactions based on bibliographic information. Subsequently, we formulated the gene-protein-reaction (GPR) relationships and rectified the model inconsistencies, including elemental, charge imbalances. We also used the amino acid auxotrophy data available in literature to manually curate the reconstructions by reducing the inconsistencies in auxotrophies observed in experiments and during constraintbased simulations. The fermentable substrate phenotyping data for 14 different carbohydrates was then used to test the agreement between model predictions and experiments, subsequently eliminating the inconsistencies through the addition of relevant metabolic and transport reactions. Growth and non-growth associated ATP maintenance costs were either adopted from existing LAB genome-scale metabolic models or estimated based on available culture data (Supplementary Dataset S3). Finally, the reconstructed models' quality and consistency were evaluated using online tool MEMOTE ${ }^{103}$ (Supplementary Dataset S4)

Existing GEMs were updated by first identifying the network gaps using gapFind algorithm ${ }^{102}$ and their subsequent resolution by adding new reactions based on bibliographic information. Next, we included new genes, reactions and metabolites and updated the GPR for several existing reactions based on the latest genome annotations, following the standard procedures ${ }^{44}$. Finally, similar to the new GEMs, we also used the amino acid auxotrophy data available in literature to manually curate the reconstructions and evaluated the model quality and consistency using MEMOTE.

\section{Constraint-based flux analysis with macromolecular crowding constraints}

Constraint-based flux analysis with macromolecular crowding constraints ${ }^{45}$ was used to analyze the metabolic phenotype of the LAB under various environmental conditions such as 
LABDM and different dietary regimes. The corresponding optimization problem can be mathematically represented as follows:

$$
\begin{gathered}
\max _{Z_{1}}=\sum_{j} c_{j} f_{j} \\
\text { subject to } \sum_{\mathrm{j}} S_{i j} f_{j}=0 \forall \text { metabolite } i \\
f_{j}^{\min } \leq f_{j} \leq f_{j}^{\max } \forall \text { reaction } j \\
\sum_{j} a_{j} f_{j} \leq 1
\end{gathered}
$$

where $Z$ is the cellular objective, $c_{j}$ is the relative weights of each metabolic reaction to biomass formation. $S_{i j}$ is the stoichiometric coefficient of metabolite $i$ of reaction $j ; f_{j}$ is the flux through the reaction $j ; f_{j}^{\min }$ and $f_{j}^{\max }$ are lower and upper bounds on the flux through the reaction $j$, respectively; and $a_{j}$ is the 'crowding coefficient' which need to be calculated based enzyme kinetic parameters, i.e. turnover numbers, and cytoplasmic density (see Supplementary Methods).

In order to simulate the physiology of exponentially growing $L A B$ in LABDM, the biomass reaction was maximized while simultaneously constraining the uptake/secretion rates of glucose and all 20 amino acids at experimentally measured values. Similarly, the exchange reactions were constrained at corresponding uptake rates provided in $\mathrm{VMH}$ database ${ }^{104}$ for simulating the LAB growth phenotype under various diet conditions. Note that we used 5000 different permutations in each simulation to account for reactions with unknown $\mathrm{C}$ values and obtained 5000 flux solutions. The geometric mean of the flux solutions surrounding the maxima of the resultant lognormal distributions accurately represent the average cellular state $^{105}$, and were then used to assess the phenotype (growth rate/byproduct secretion) of the LAB (see Supplementary Results and Supplementary Figure S5). All simulations were implemented using COBRA toolbox ${ }^{106}$ and Gurobi7 (http://www.gurobi.com) optimization solver (see Supplementary Methods for more details). 


\section{Simulation of postbiotic production potential in $L A B$}

In order to compute the theoretical yield of the postbiotic metabolites, pseudo-reactions representing their secretion was added and iteratively used as the objective function. Biomass was constrained to $50 \%$ of the optimum value. FBAwMC was implemented using COBRA toolbox ${ }^{108}$ functionalities and the objective was maximized to obtain the in silico postbiotic yields of each LAB while constraining the exchange reactions at corresponding uptake rates provided in $\mathrm{VMH}$ database for each diet. We used 5000 different permutations for each FBAwMC simulation to account for reactions with unknown $C$ values. The geometric mean of the resulting 5000 flux solutions surrounding the maxima of the lognormal distributions accurately represents the average cellular state ${ }^{105}$, and was then used to calculate the postbiotic yield. Finally, the yield of each postbiotic compound was normalized to the maximum value across different diets and LAB.

\section{In silico examination of pairwise interactions between $L A B$ and other microbes}

Pairwise microbial interactions between LAB and any other microbe under anaerobic conditions were evaluated in silico based a method proposed earlier ${ }^{109}$. Briefly, the LAB model was first paired with any of other microbe $\mathrm{GEMs}^{110-12}>$ by introducing a common lumen compartment to facilitate the exchange of nutrients between them. Second, the exchange reactions of various compounds in each diets were added to the lumen.

The pairwise interactions were then evaluated using the enzyme-capacity constrained paired model as follows. First, the biomass of both species in the paired model was maximized under a particular diet or $\mathrm{BHI}$ media to obtain the growth rates of individual species when they grow together. Next, the maximum growth of individual species when they grow in isolation under the same diet was computed while simultaneously constraining all the reactions of other species to zero. Finally, by comparing the growth rates of paired and isolation conditions, the pairwise interactions are classified as follows: i) competition - if both organisms grow slower in paired condition than isolated conditions, ii) parasitism - one species grows faster while other grows slower in paired condition than isolated conditions, iii) mutualism - both species 
bioRxiv preprint doi: https://doi.org/10.1101/2021.06.20.449192· this version posted June 21 2021. The copyright holder for this preprint (which was not certified by peer review) is the author/funder, who has granted bioRxiv a license to display the preprint in perpetuity. It is made available under aCC-BY-NC-ND 4.0 International license.

grow faster in paired condition than isolated conditions, iv) amensalism - one species grow slower while the other is unaffected in paired condition than isolated conditions, v) commensalism - one species grow faster while the other is unaffected in paired condition than isolated conditions and vi) neutralism - no appreciable difference in both species growths between paired and isolated conditions. Note that a species was considered to grow faster in the paired condition when its growth rate was more than $10 \%$ higher than isolated condition. The exchange reactions were constrained at corresponding uptake rates provided in $\mathrm{VMH}$ database ${ }^{104}$ or values calculated based on a previous study ${ }^{121}$ for simulating the LAB growth phenotype under various diet conditions or $\mathrm{BHI}$ media, respectively. All pairwise interactions were simulated using microbiome modeling toolbox ${ }^{122}$.

\section{References}

1. Holzapfel, W. H. \& Wood, B. J. B. Lactic Acid Bacteria: Biodiversity and Taxonomy. in Lactic Acid Bacteria: Biodiversity and Taxonomy (2014). doi:10.1002/9781118655252

2. Gänzle, M. G. Lactic metabolism revisited: Metabolism of lactic acid bacteria in food fermentations and food spoilage. Curr. Opin. Food Sci. 2, 106-117 (2015).

3. Carr, F. J., Chill, D. \& Maida, N. The lactic acid bacteria: A literature survey. Crit. Rev. Microbiol. 28, 281-370 (2002).

4. Golić, N. et al. In vitro and in vivo antagonistic activity of new probiotic culture against Clostridium difficile and Clostridium perfringens. BMC Microbiol. 17, 108 (2017).

5. Shelby, R. D. et al. A novel probiotic therapeutic in a murine model of Clostridioides difficile colitis. Gut Microbes 12, 1814119 (2020).

6. Ma, Y. et al. Which probiotic has the best effect on preventing Clostridium difficileassociated diarrhea? A systematic review and network meta-analysis. J. Dig. Dis. 21, 69-80 (2020).

7. Nakazato, G. et al. Lactobacillus acidophilus decreases salmonella typhimurium invasion in vivo. J. Food Saf. 31, 284-289 (2011).

8. Maldonado Galdeano, C. \& Perdigón, G. The probiotic bacterium Lactobacillus casei induces activation of the gut mucosal immune system through innate immunity. Clin. Vaccine Immunol. 13, 219-226 (2006).

9. Garcia-Castillo, V. et al. Evaluation of the immunomodulatory activities of the probiotic strain lactobacillus fermentum UCO-979C. Front. Immunol. 10, 1376 (2019).

10. Zhang, D., Li, C., Shi, R., Zhao, F. \& Yang, Z. Lactobacillus fermentum JX306 restrain d-galactose-induced oxidative stress of mice through its antioxidant activity. Polish $\mathrm{J}$. Microbiol. 69, 205-215 (2020).

11. Zhao, J. et al. Lactobacillus plantarum CCFM10 alleviating oxidative stress and 
restoring the gut microbiota in d-galactose-induced aging mice. Food Funct. 9, 917924 (2018).

12. Toi, M. et al. Probiotic Beverage with Soy Isoflavone Consumption for Breast Cancer Prevention: A Case-control Study. Curr. Nutr. Food Sci. 9, 194-200 (2013).

13. El-Nezami, H. S. et al. Probiotic supplementation reduces a biomarker for increased risk of liver cancer in young men from Southern China. Am. J. Clin. Nutr. 83, 11991203 (2006).

14. Hardy, H., Harris, J., Lyon, E., Beal, J. \& Foey, A. D. Probiotics, prebiotics and immunomodulation of gut mucosal defences: Homeostasis and immunopathology. Nutrients 5, 1869-1912 (2013).

15. van Baarlen, P., Wells, J. M. \& Kleerebezem, M. Regulation of intestinal homeostasis and immunity with probiotic lactobacilli. Trends Immunol. 34, 208-215 (2013).

16. Azas-Braesco, V., Bresson, J. L., Guarner, F. \& Corthier, G. Not all lactic acid bacteria are probiotics, but some are. Br. J. Nutr. 103, 1079-81 (2010).

17. Ibnou-Zekri, N., Blum, S., Schiffrin, E. J. \& Von der Weid, T. Divergent patterns of colonization and immune response elicited from two intestinal Lactobacillus strains that display similar properties in vitro. Infect. Immun. 71, 428-436 (2003).

18. Campana, R., van Hemert, S. \& Baffone, W. Strain-specific probiotic properties of lactic acid bacteria and their interference with human intestinal pathogens invasion. Gut Pathog. 9, 1-12 (2017).

19. Liu, J. et al. Strain-specific properties of Lactobacillus plantarum for prevention of Salmonella infection. Food Funct. 9, 3673-3682 (2018).

20. Dietrich, C. G., Kottmann, T. \& Alavi, M. Commercially available probiotic drinks containing Lactobacillus casei DN-114001 reduce antibiotic-associated diarrhea. World J. Gastroenterol. 20, 15837-44 (2014).

21. De Roock, S. et al. Lactic acid bacteria differ in their ability to induce functional regulatory T cells in humans. Clin. Exp. Allergy 40, 103-10 (2010).

22. Ashraf, R., Vasiljevic, T., Day, S. L., Smith, S. C. \& Donkor, O. N. Lactic acid bacteria and probiotic organisms induce different cytokine profile and regulatory $\mathrm{T}$ cells mechanisms. J. Funct. Foods 6, 395-409 (2014).

23. Xia, Y. et al. Effects of dietary Lactobacillus rhamnosus JCM1136 and Lactococcus lactis subsp. lactis JCM5805 on the growth, intestinal microbiota, morphology, immune response and disease resistance of juvenile Nile tilapia, Oreochromis niloticus. Fish Shellfish Immunol. 76, 368-379 (2018).

24. Møller, P. L., Paerregaard, A., Gad, M., Kristensen, N. N. \& Claesson, M. H. Colitic scid mice fed Lactobacillus spp. show an ameliorated gut histopathology and an altered cytokine profile by local T cells. Inflamm. Bowel Dis. 11, 814-9 (2005).

25. D'Arienzo, R. et al. Distinct immunomodulatory properties of Lactobacillus paracasei strains. J. Appl. Microbiol. 111, 1482-1491 (2011).

26. Nerstedt, A. et al. Administration of Lactobacillus evokes coordinated changes in the intestinal expression profile of genes regulating energy homeostasis and immune phenotype in mice. Br. J. Nutr. 97, 1117-1127 (2007).

27. Martín, R. et al. Probiotic potential of 3 lactobacilli strains isolated from breast milk. J. Hum. Lact. 21, 8-17 (2005). 
bioRxiv preprint doi: https://doi org/10.1101/2021.06.20 449192: this version posted June 21 2021. The copyright holder for this preprint (which was not certified by peer review) is the author/funder, who has granted bioRxiv a license to display the preprint in perpetuity. It is made available under aCC-BY-NC-ND 4.0 International license.

28. Ruiz, L., Margolles, A. \& Sánchez, B. Bile resistance mechanisms in Lactobacillus and Bifidobacterium. Front. Microbiol. 4, 396 (2013).

29. Zhou, Z. et al. Identification of highly-adhesive gut Lactobacillus strains in zebrafish (Danio rerio) by partial rpoB gene sequence analysis. Aquaculture 370-371, 150-157 (2012).

30. Pagnini, C. et al. Mucosal adhesion and anti-inflammatory effects of Lactobacillus rhamnosus GG in the human colonic mucosa: A proof-of-concept study. World J. Gastroenterol. 24, 4652-4662 (2018).

31. Turpin, W., Humblot, C., Noordine, M. L., Thomas, M. \& Guyot, J. P. Lactobacillaceae and cell adhesion: Genomic and functional screening. PLoS One 7, e38034 (2012).

32. Wu, C., Huang, J. \& Zhou, R. Genomics of lactic acid bacteria: Current status and potential applications. Crit. Rev. Microbiol. 43, 393-404 (2017).

33. De Filippis, F., Pasolli, E. \& Ercolini, D. The food-gut axis: Lactic acid bacteria and their link to food, the gut microbiome and human health. FEMS Microbiol. Rev. 44, 454-489 (2020).

34. Makarova, K. S. et al. Comparative genomics of the lactic acid bacteria. Proc. Natl. Acad. Sci. U. S. A. 103, 15611-15616 (2006).

35. Sun, Z. et al. Expanding the biotechnology potential of lactobacilli through comparative genomics of 213 strains and associated genera. Nat. Commun. 6, 8322 (2015).

36. Zhang, G., Mills, D. A. \& Block, D. E. Development of chemically defined media supporting high-cell-density growth of lactococci, enterococci, and streptococci. Appl. Environ. Microbiol. 75, 1080-1087 (2009).

37. Koduru, L. et al. Genome-scale modeling and transcriptome analysis of Leuconostoc mesenteroides unravel the redox governed metabolic states in obligate heterofermentative lactic acid bacteria. Sci. Rep. 7, 15721 (2017).

38. Pasolli, E. et al. Large-scale genome-wide analysis links lactic acid bacteria from food with the gut microbiome. Nat. Commun. 11, 2610 (2020).

39. Valeriano, V. D. V., Oh, J. K., Bagon, B. B., Kim, H. \& Kang, D. K. Comparative genomic analysis of Lactobacillus mucosae LM1 identifies potential niche-specific genes and pathways for gastrointestinal adaptation. Genomics 111, (2019).

40. Drissi, F. et al. Comparative genomics analysis of Lactobacillus species associated with weight gain or weight protection. Nutr. Diabetes 4, e109 (2014).

41. Klaenhammer, T. R., Barrangou, R., Buck, B. L., Azcarate-Peril, M. A. \& Altermann, E. Genomic features of lactic acid bacteria effecting bioprocessing and health. FEMS Microbiol. Rev. 29, 393-409 (2005).

42. Teusink, B. et al. Analysis of growth of Lactobacillus plantarum WCFS1 on a complex medium using a genome-scale metabolic model. J. Biol. Chem. 281, 40041-40048 (2006).

43. Flahaut, N. A. L. et al. Genome-scale metabolic model for Lactococcus lactis MG1363 and its application to the analysis of flavor formation. Appl. Microbiol. Biotechnol. 97, 8729-8739 (2013).

44. Thiele, I. \& Palsson, B. Ø. A protocol for generating a high-quality genome-scale metabolic reconstruction. Nat. Protoc. 5, 93-121 (2010).

45. Beg, Q. K. et al. Intracellular crowding defines the mode and sequence of substrate 
uptake by Escherichia coli and constrains its metabolic activity. Proc. Natl. Acad. Sci. U. S. A. 104, 12663-8 (2007).

46. Vazquez, A., Liu, J., Zhou, Y. \& Oltvai, Z. N. Catabolic efficiency of aerobic glycolysis: the Warburg effect revisited. BMC Syst. Biol. 4, 58 (2010).

47. Shlomi, T., Benyamini, T., Gottlieb, E., Sharan, R. \& Ruppin, E. Genome-scale metabolic modeling elucidates the role of proliferative adaptation in causing the warburg effect. PLoS Comput. Biol. 7, e1002018 (2011).

48. van Hoek, M. J. A. \& Merks, R. M. H. Redox balance is key to explaining full vs. partial switching to low-yield metabolism. BMC Syst. Biol. 6, 22 (2012).

49. Oliveira, A. P., Nielsen, J. \& Förster, J. Modeling Lactococcus lactis using a genomescale flux model. BMC Microbiol. 5, 39 (2005).

50. Wu, G. D. et al. Linking long-term dietary patterns with gut microbial enterotypes. Sci. (New York, NY) 334, 105-8 (2011).

51. David, L. A. et al. Diet rapidly and reproducibly alters the human gut microbiome. Nature 505, 559-563 (2014).

52. Zarrinpar, A., Chaix, A., Yooseph, S. \& Panda, S. Diet and feeding pattern affect the diurnal dynamics of the gut microbiome. Cell Metab. 20, 1006-17 (2014).

53. Gänzle, M. G. \& Follador, R. Metabolism of oligosaccharides and starch in lactobacilli: A review. Front. Microbiol. 3, 1-15 (2012).

54. Yao, J. \& Rock, C. O. Exogenous fatty acid metabolism in bacteria. Biochimie 141, 3039 (2017).

55. Suez, J., Zmora, N., Segal, E. \& Elinav, E. The pros, cons, and many unknowns of probiotics. Nat. Med. 25, 716-729 (2019).

56. Vlasova, A. N. et al. Lactobacilli and Bifidobacteria Promote Immune Homeostasis by Modulating Innate Immune Responses to Human Rotavirus in Neonatal Gnotobiotic Pigs. PLoS One 8, e76962 (2013).

57. Pavan, S., Desreumaux, P. \& Mercenier, A. Use of mouse models to evaluate the persistence, safety, and immune modulation capacities of lactic acid bacteria. Clin. Diagn. Lab. Immunol. 10, 696-701 (2003).

58. Trindade, S. et al. Positive epistasis drives the acquisition of multidrug resistance. PLoS Genet. 5, e1000578 (2009).

59. Silva, R. F. et al. Pervasive sign epistasis between conjugative plasmids and DrugResistance chromosomal mutations. PLoS Genet. 7, e1002181 (2011).

60. Khan, A. I., Dinh, D. M., Schneider, D., Lenski, R. E. \& Cooper, T. F. Negative epistasis between beneficial mutations in an evolving bacterial population. Sci. (New York, NY) 332, 1193-1196 (2011).

61. Zadeh, M. et al. Induction of intestinal pro-inflammatory immune responses by lipoteichoic acid. J. Inflamm. 9, 7 (2012).

62. Mendes, M. C. S. et al. Microbiota modification by probiotic supplementation reduces colitis associated colon cancer in mice. World J. Gastroenterol. 24, 1995-2008 (2018).

63. Shin, D. et al. Beneficial roles of probiotics on the modulation of gut microbiota and immune response in pigs. PLoS One 14, e0220843 (2019).

64. Lebas, M., Garault, P., Carrillo, D., Codoñer, F. M. \& Derrien, M. Metabolic response of 
Faecalibacterium prausnitzii to cell-free supernatants from lactic acid bacteria. Microorganisms 8, 1-15 (2020).

65. El Hage, R., Hernandez-Sanabria, E. \& Van de Wiele, T. Emerging trends in 'smart probiotics': Functional consideration for the development of novel health and industrial applications. Front. Microbiol. 8, 1889 (2017).

66. de Melo Pereira, G. V., de Oliveira Coelho, B., Magalhães Júnior, A. I., Thomaz-Soccol, V. \& Soccol, C. R. How to select a probiotic? A review and update of methods and criteria. Biotechnol. Adv. 36, 2060-2076 (2018).

67. Wan, Y. et al. Effects of dietary fat on gut microbiota and faecal metabolites, and their relationship with cardiometabolic risk factors: A 6-month randomised controlled-feeding trial. Gut 68, 1417-1429 (2019).

68. Swidsinski, A. et al. Reduced mass and diversity of the colonic microbiome in patients with multiple sclerosis and their improvement with ketogenic diet. Front. Microbiol. 8, 1141 (2017).

69. Brinkworth, G. D., Noakes, M., Clifton, P. M. \& Bird, A. R. Comparative effects of very low-carbohydrate, high-fat and high-carbohydrate, low-fat weight-loss diets on bowel habit and faecal short-chain fatty acids and bacterial populations. Br. J. Nutr. 101, 1493-502 (2009).

70. Lopez-Siles, M. et al. Alterations in the Abundance and Co-occurrence of Akkermansia muciniphila and Faecalibacterium prausnitzii in the Colonic Mucosa of Inflammatory Bowel Disease Subjects. Front. Cell. Infect. Microbiol. 8, 281 (2018).

71. Lenoir, M. et al. Lactobacillus casei BL23 regulates Treg and Th17 T-cell populations and reduces DMH-associated colorectal cancer. J. Gastroenterol. 51, 862-73 (2016).

72. Yan, F. et al. Soluble Proteins Produced by Probiotic Bacteria Regulate Intestinal Epithelial Cell Survival and Growth. Gastroenterology 132, 562-75 (2007).

73. Hor, Y. Y. et al. Probiotic Lactobacillus casei Zhang (LCZ) alleviates respiratory, gastrointestinal \& RBC abnormality via immuno-modulatory, anti-inflammatory \& antioxidative actions. J. Funct. Foods 44, 235-245 (2018).

74. Tien, M.-T. et al. Anti-inflammatory effect of Lactobacillus casei on Shigella-infected human intestinal epithelial cells. J. Immunol. 176, 1228-37 (2006).

75. Vaghef-Mehrabany, E. et al. Probiotic supplementation improves inflammatory status in patients with rheumatoid arthritis. Nutrition 3, 430-5 (2014).

76. Schiffer, C. et al. A Strain of Lactobacillus casei Inhibits the Effector Phase of Immune Inflammation. J. Immunol. 187, 2646-55 (2011).

77. Taur, Y. \& Pamer, E. G. The intestinal microbiota and susceptibility to infection in immunocompromised patients. Curr. Opin. Infect. Dis. 26, 332-7 (2013).

78. Aktas, B., De Wolfe, T. J., Safdar, N., Darien, B. J. \& Steele, J. L. The impact of Lactobacillus casei on the composition of the cecal microbiota and innate immune system is strain specific. PLoS One 11, e0156374 (2016).

79. Lee, I. C. et al. Strain-specific features of extracellular polysaccharides and their impact on Lactobacillus plantarum-host interactions. Appl. Environ. Microbiol. 82, 3959-3970 (2016).

80. Kolling, Y., Salva, S., Villena, J. \& Alvarez, S. Are the immunomodulatory properties of Lactobacillus rhamnosus CRL1505 peptidoglycan common for all Lactobacilli during respiratory infection in malnourished mice? PLoS One 13, e0194034 (2018). 
81. Bradford, M. M. A rapid and sensitive method for the quantitation of microgram quantities of protein utilizing the principle of protein-dye binding. Anal. Biochem. (1976). doi:10.1016/0003-2697(76)90527-3

82. Sprouffske, K. \& Wagner, A. Growthcurver: An R package for obtaining interpretable metrics from microbial growth curves. BMC Bioinformatics 17, 172 (2016).

83. Meier-Kolthoff, J. P. \& Göker, M. TYGS is an automated high-throughput platform for state-of-the-art genome-based taxonomy. Nat. Commun. 10, 2182 (2019).

84. Meier-Kolthoff, J. P., Auch, A. F., Klenk, H. P. \& Göker, M. Genome sequence-based species delimitation with confidence intervals and improved distance functions. BMC Bioinformatics 14, 60 (2013).

85. Lefort, V., Desper, R. \& Gascuel, O. FastME 2.0: A comprehensive, accurate, and fast distance-based phylogeny inference program. Mol. Biol. Evol. 32, 2798-2800 (2015).

86. Letunic, I. \& Bork, P. Interactive Tree of Life (iTOL) v4: Recent updates and new developments. Nucleic Acids Res. 44, W242-W245 (2019).

87. Sonnhammer, E. L. L. \& Östlund, G. InParanoid 8: Orthology analysis between 273 proteomes, mostly eukaryotic. Nucleic Acids Res. 43, D234-D239 (2015).

88. Huerta-Cepas, J. et al. EggNOG 5.0: A hierarchical, functionally and phylogenetically annotated orthology resource based on 5090 organisms and 2502 viruses. Nucleic Acids Res. (2019). doi:10.1093/nar/gky1085

89. Moreno-Hagelsieb, G. \& Latimer, K. Choosing BLAST options for better detection of orthologs as reciprocal best hits. Bioinformatics 24, 319-324 (2008).

90. de Jong, A., van Heel, A. J., Kok, J. \& Kuipers, O. P. BAGEL2: Mining for bacteriocins in genomic data. Nucleic Acids Res. 38, W647-W651 (2010).

91. Andrews, S. FASTQC A Quality Control tool for High Throughput Sequence Data. Babraham Inst. (2015).

92. Bolger, A. M., Lohse, M. \& Usadel, B. Trimmomatic: a flexible trimmer for Illumina sequence data. Bioinformatics 30, 2114-20 (2014).

93. Dobin, A. et al. STAR: ultrafast universal RNA-seq aligner. Bioinformatics 29, 15-21 (2013).

94. Li, B. \& Dewey, C. N. RSEM: accurate transcript quantification from RNA-Seq data with or without a reference genome. BMC Bioinformatics 12, 323 (2011).

95. Benjamini, Y. \& Hochberg, Y. Controlling the False Discovery Rate: A Practical and Powerful Approach to Multiple Testing. J. R. Stat. Soc. Ser. B 57, 289-300 (1995).

96. Peixoto, L. et al. How data analysis affects power, reproducibility and biological insight of RNA-seq studies in complex datasets. Nucleic Acids Res. 43, 7664-7674 (2015).

97. Love, M. I., Huber, W. \& Anders, S. Moderated estimation of fold change and dispersion for RNA-seq data with DESeq2. Genome Biol. 15, 550 (2014).

98. Dennis, G. et al. DAVID: Database for Annotation, Visualization, and Integrated Discovery. Genome Biol. 4, P3 (2003).

99. Ogata, H. et al. KEGG: Kyoto encyclopedia of genes and genomes. Nucleic Acids Research 27, 29-34 (1999).

100. Caspi, R. et al. The MetaCyc database of metabolic pathways and enzymes and the BioCyc collection of Pathway/Genome Databases. Nucleic Acids Res. 42, (2014). 
bioRxiv preprint doi: https://doi org/10.1101/2021.06.20 449192: this version posted June 21,2021 . The copyright holder for this preprint (which was not certified by peer review) is the author/funder, who has granted bioRxiv a license to display the preprint in perpetuity. It is made available under aCC-BY-NC-ND 4.0 International license.

101. Feist, A. M. \& Palsson, B. O. The biomass objective function. Curr. Opin. Microbiol. 13, 344-349 (2010).

102. Kumar, V. S. \& Maranas, C. D. GrowMatch: an automated method for reconciling in silico/in vivo growth predictions. PLoS Comput. Biol. 5, e1000308 (2009).

103. Lieven, C. et al. Memote: A community driven effort towards a standardized genomescale metabolic model test suite. bioRxiv (2018). doi:10.1101/350991

104. Noronha, A. et al. The Virtual Metabolic Human database: Integrating human and gut microbiome metabolism with nutrition and disease. Nucleic Acids Res. 47, D614-D624 (2019).

105. Bengtsson, M., Ståhlberg, A., Rorsman, P. \& Kubista, M. Gene expression profiling in single cells from the pancreatic islets of Langerhans reveals lognormal distribution of mRNA levels. Genome Res. 15, 1388-1392 (2005).

106. Schellenberger, J. et al. Quantitative prediction of cellular metabolism with constraintbased models: the COBRA Toolbox v2.0. Nat. Protoc. 6, 1290-307 (2011).

107. Segrè, D., Deluna, A., Church, G. M. \& Kishony, R. Modular epistasis in yeast metabolism. Nat. Genet. 37, 77-83 (2005).

108. Heirendt, L. et al. Creation and analysis of biochemical constraint-based models using the COBRA Toolbox v.3.0. Nat. Protoc. 14, 639-702 (2019).

109. Magnúsdóttir, S. et al. Generation of genome-scale metabolic reconstructions for 773 members of the human gut microbiota. Nat. Biotechnol. 35, 81-89 (2017).

110. Monk, J. M. et al. Genome-scale metabolic reconstructions of multiple Escherichia coli strains highlight strain-specific adaptations to nutritional environments. Proc. Natl. Acad. Sci. U. S. A. 110, 20338-20343 (2013).

111. Bosi, E. et al. Comparative genome-scale modelling of Staphylococcus aureus strains identifies strain-specific metabolic capabilities linked to pathogenicity. Proc. Natl. Acad. Sci. U. S. A. 113, E3801-E3809 (2016).

112. Liao, Y.-C. et al. An experimentally validated genome-scale metabolic reconstruction of Klebsiella pneumoniae MGH 78578, iYL1228. J. Bacteriol. 193, 1710-7 (2011).

113. Raghunathan, A., Reed, J., Shin, S., Palsson, B. \& Daefler, S. Constraint-based analysis of metabolic capacity of Salmonella typhimurium during host-pathogen interaction. BMC Syst. Biol. 3, 1-16 (2009).

114. Thiele, I., Vo, T. D., Price, N. D. \& Palsson, B. Ø. Expanded Metabolic Reconstruction of Helicobacter pylori ( i IT341 GSM / GPR ): an In Silico Genome-Scale Characterization of Single- and Double-Deletion Mutants. J. Bacteriol. 187, 5818-5830 (2005).

115. Nogales, J., Palsson, B. \& Thiele, I. A genome-scale metabolic reconstruction of Pseudomonas putida KT2440: iJN746 as a cell factory. BMC Syst. Biol. 2, 79 (2008).

116. Oberhardt, M. A., Puchałka, J., Fryer, K. E., Martins dos Santos, V. A. P. \& Papin, J. A. Genome-scale metabolic network analysis of the opportunistic pathogen Pseudomonas aeruginosa PAO1. J. Bacteriol. 190, 2790-803 (2008).

117. Veith, N. et al. Using a genome-scale metabolic model of Enterococcus faecalis V583 to assess amino acid uptake and its impact on central metabolism. Appl. Environ. Microbiol. 81, 1622-1633 (2015).

118. Ottman, N. et al. Genome-scale model and omics analysis of metabolic capacities of 
Akkermansia muciniphila reveal a preferential mucin-degrading lifestyle. Appl. Environ. Microbiol. 83, e01014-17 (2017).

119. El-Semman, I. E. et al. Genome-scale metabolic reconstructions of Bifidobacterium adolescentis L2-32 and Faecalibacterium prausnitzii A2-165 and their interaction. BMC Syst. Biol. 8, 41 (2014).

120. Heinken, A., Sahoo, S., Fleming, R. M. T. \& Thiele, I. Systems-level characterization of a host-microbe metabolic symbiosis in the mammalian gut. Gut Microbes 4, 28-40 (2013).

121. Puccio, T., Misra, B. B. \& Kitten, T. Time-course analysis of Streptococcus sanguinis after manganese depletion reveals changes in glycolytic, nucleotide, and redox metabolites. bioRxiv (2020).

122. Baldini, F. et al. The Microbiome Modeling Toolbox: from microbial interactions to personalized microbial communities. Bioinformatics 35, 2332-2334 (2019).

123. Glont, M. et al. BioModels: Expanding horizons to include more modelling approaches and formats. Nucleic Acids Res. 46, D1248-D1253 (2018).

\section{Acknowledgements}

The authors thank Shawn Hoon and Fong Tian Wong for advice on RNA-seq library preparation steps, Elena Heng for experimental assistance in transcriptome sequencing, and Say Kong Ng and Tessa Tan for their advice and support on HPLC analysis.

\section{Author contributions}

L.K., M.L., D.S.-W.O. and D.-Y.L. conceived the project. L.K., P.-Y.L. and M.B. performed LAB experiments, and profiled culture supernatants. P. L. H. and D.-S. P performed commensal culture experiments. L.K. formulated the new LABDM with inputs from D.S.-W.O. L.K., P.-Y.L. and M.L. were involved in transcriptome sequencing, assembly, and relevant bioinformatics analysis. L.K. and M.L. performed the comparative genomic analyses. L.K. and M.L. reconstructed the genome-scale models, developed in silico methods and implemented them. L.K. and M.L. drafted the initial manuscript. L.K., M.L., D.S.-W.O. and D.-Y.L. were involved in editing and revising the manuscript. D.S.-W.O. and D.-Y.L. supervised and coordinated the project. 
bioRxiv preprint doi: https:/doi.org/10.1101/2021.06.20.449192; this version posted June 21,2021 . The copyright holder for this preprint (which was not certified by peer review) is the author/funder, who has granted bioRxiv a license to display the preprint in perpetuity. It is made available under aCC-BY-NC-ND 4.0 International license.

\section{Figures}

a

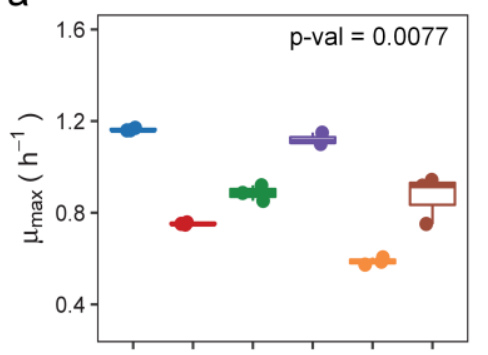

b

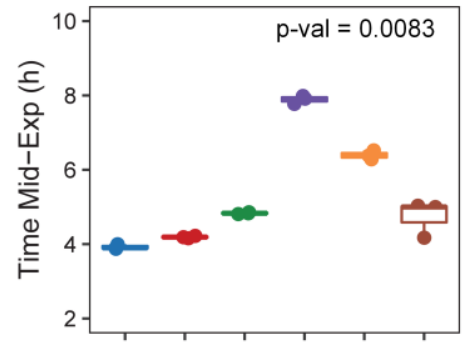

C

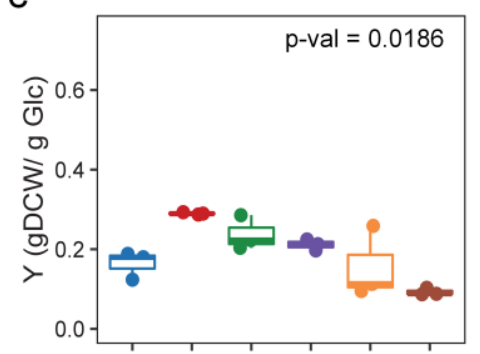

d

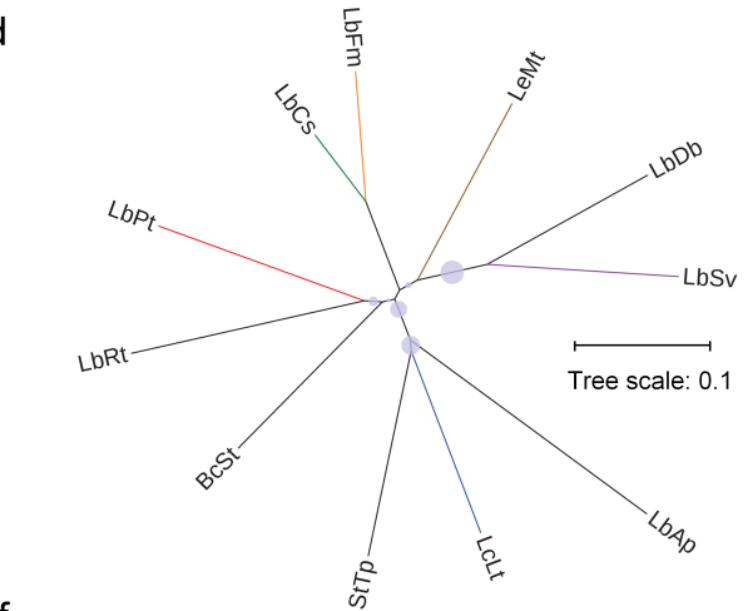

f

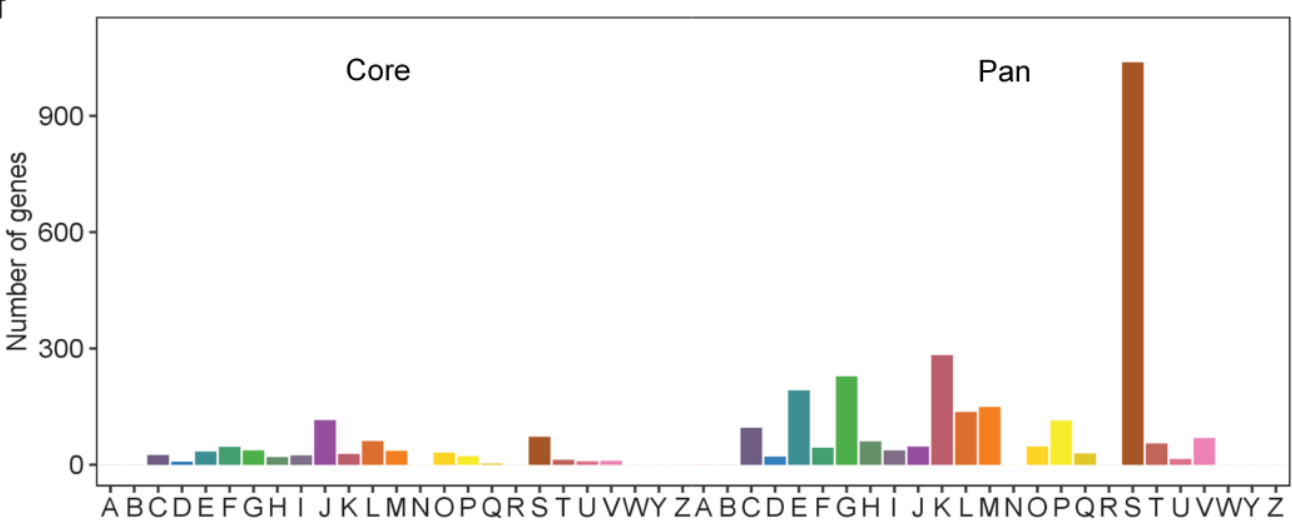

g

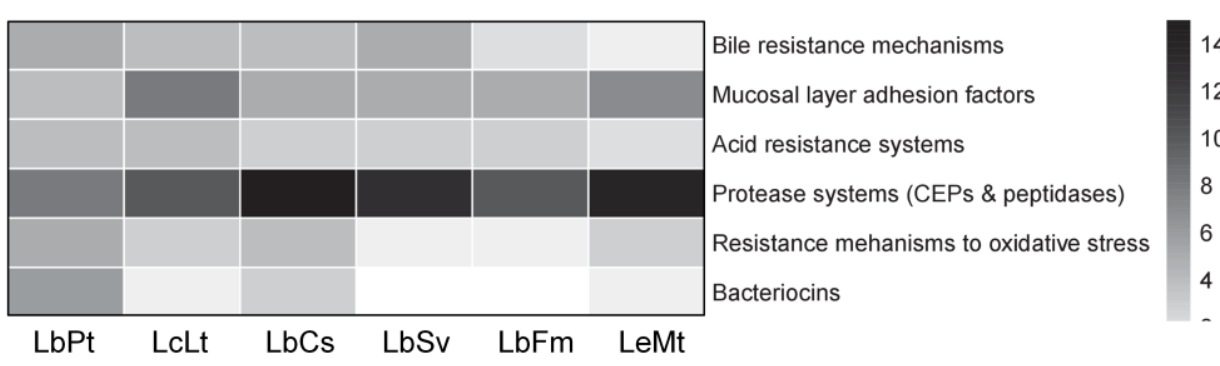

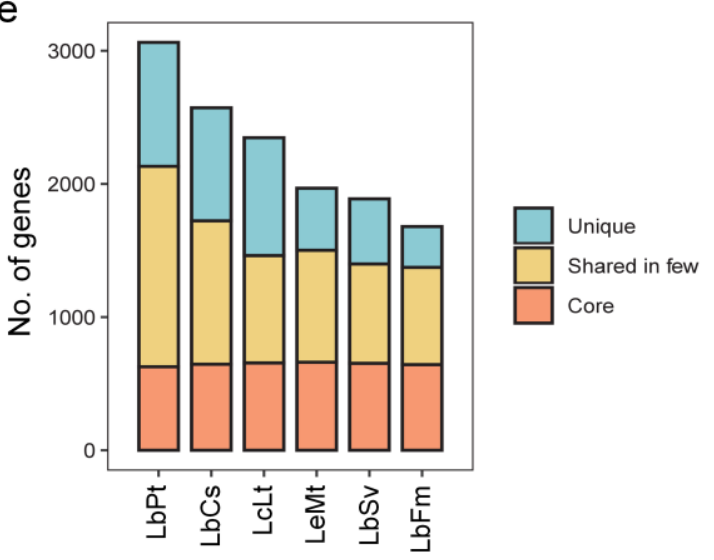

COG category

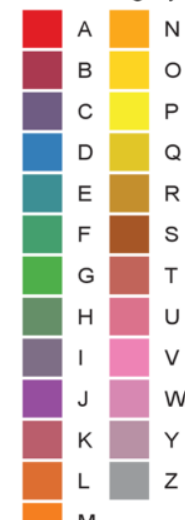

No. of genes

Figure 1. Growth phenotype and genomic characteristics of LAB strains. (a) Differences in the maximum growth rates across $L A B$ in LABDM cultures ( $p$-val $=0.0077$, Kruskal-Wallis test), (b) differences in time required to reach mid-exponential phase across LAB in LABDM cultures ( $p$-val = 0.0083 , Kruskal-Wallis test), (c) Differences in yield of biomass over glucose across LAB in LABDM cultures ( $p$-val $=0.0186$, Kruskal-Wallis test), (d) phylogenetic tree showing the relationship between 
bioRxiv preprint doi: https://doi.org/10.1101/2021.06.20.449192; this version posted June 21, 2021. The copyright holder for this preprint (which was not certified by peer review) is the author/funder, who has granted bioRxiv a license to display the preprint in perpetuity. It is made available under aCC-BY-NC-ND 4.0 International license.

several $L A B$ species with an outgroup member $B$. subtilis (BaSt), (e) the number of core and pan genes in LAB strains, (f) the functional annotation of core and pan genes using NOG mappings, (g) comparison of major non-metabolic genetic determinants influencing probiotic efficacy of LAB. Boxes in (a-c) include 1 st to 3rd quartile of each population while whiskers extend up to 1.5 times the interquartile range from the closest box boundary. The size of circles in (d) represent the bootstrap values. Core genes in (f) are the genes which is present in all 6 LAB. Pan genes include both unique genes and the ones which ortholog only in a few. NOG categories in (f) is as follows: A - RNA processing and modification, B Chromatin structure and dynamics, C - Energy production and conversion, D - Cell cycle control, cell division, chromosome partitioning, E - Amino acid transport and metabolism, F - Nucleotide transport and metabolism, $\mathrm{G}$ - Carbohydrate transport and metabolism, $\mathrm{H}$ - Coenzyme transport and metabolism, $\mathrm{I}$ - Lipid transport and metabolism, J - Translation, ribosomal structure and biogenesis, $\mathrm{K}$ Transcription, $\mathrm{L}-$ Replication, recombination and repair, $\mathrm{M}$ - Cell wall/membrane/envelope biogenesis, $\mathrm{N}$ - Cell motility, O - Posttranslational modification, protein turnover, chaperones, P - Inorganic ion transport and metabolism, Q - Secondary metabolites biosynthesis, transport and catabolism, R General function prediction only, S - Function unknown, T - Signal transduction mechanisms, U Intracellular trafficking, secretion, and vesicular transport, $\mathrm{V}$ - Defense mechanisms, $\mathrm{W}$ - Extracellular structures, $\mathrm{Y}$ - Nuclear structure and Z - Cytoskeleton. 
bioRxiv preprint doi: https://doi.org/10.1101/2021.06.20.449192; this version posted June 21, 2021. The copyright holder for this preprint (which was not certified by peer review) is the author/funder, who has granted bioRxiv a license to display the preprint in perpetuity. It is made available under aCC-BY-NC-ND 4.0 International license.
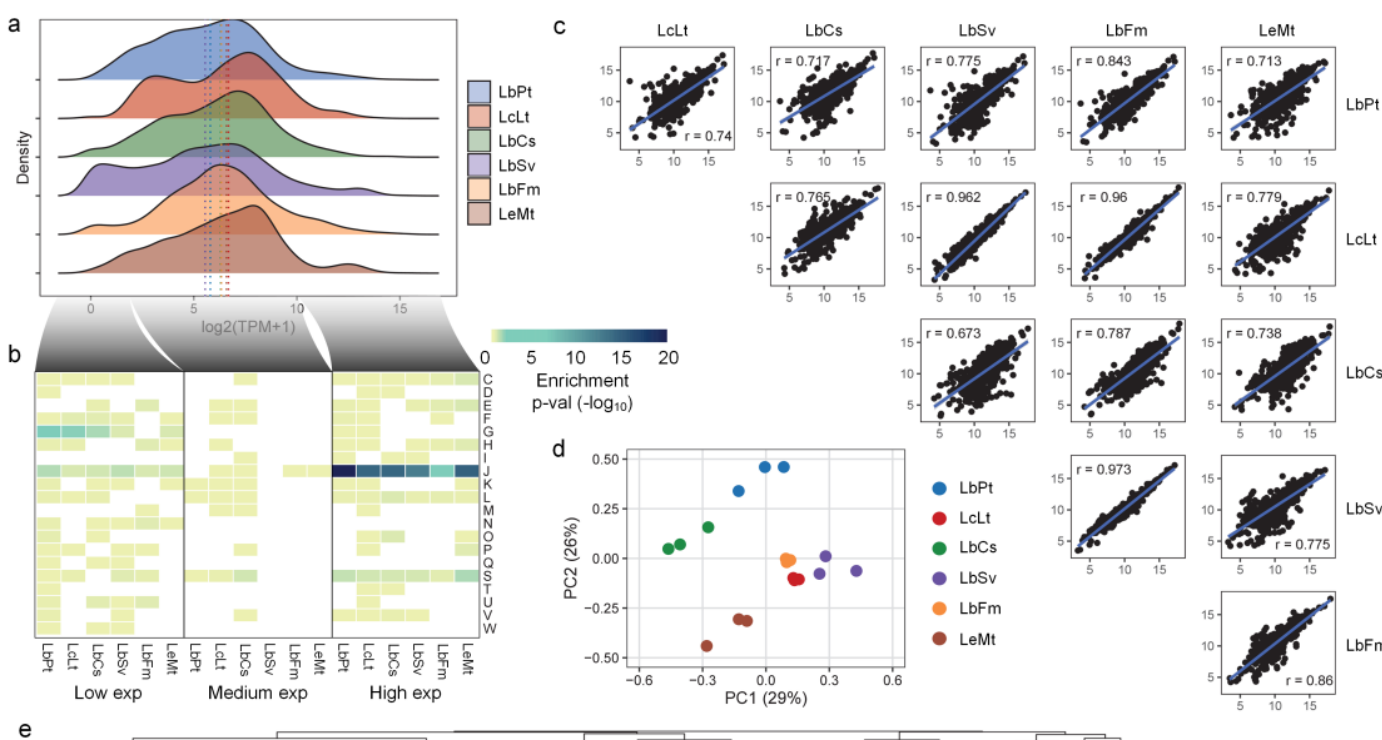

b
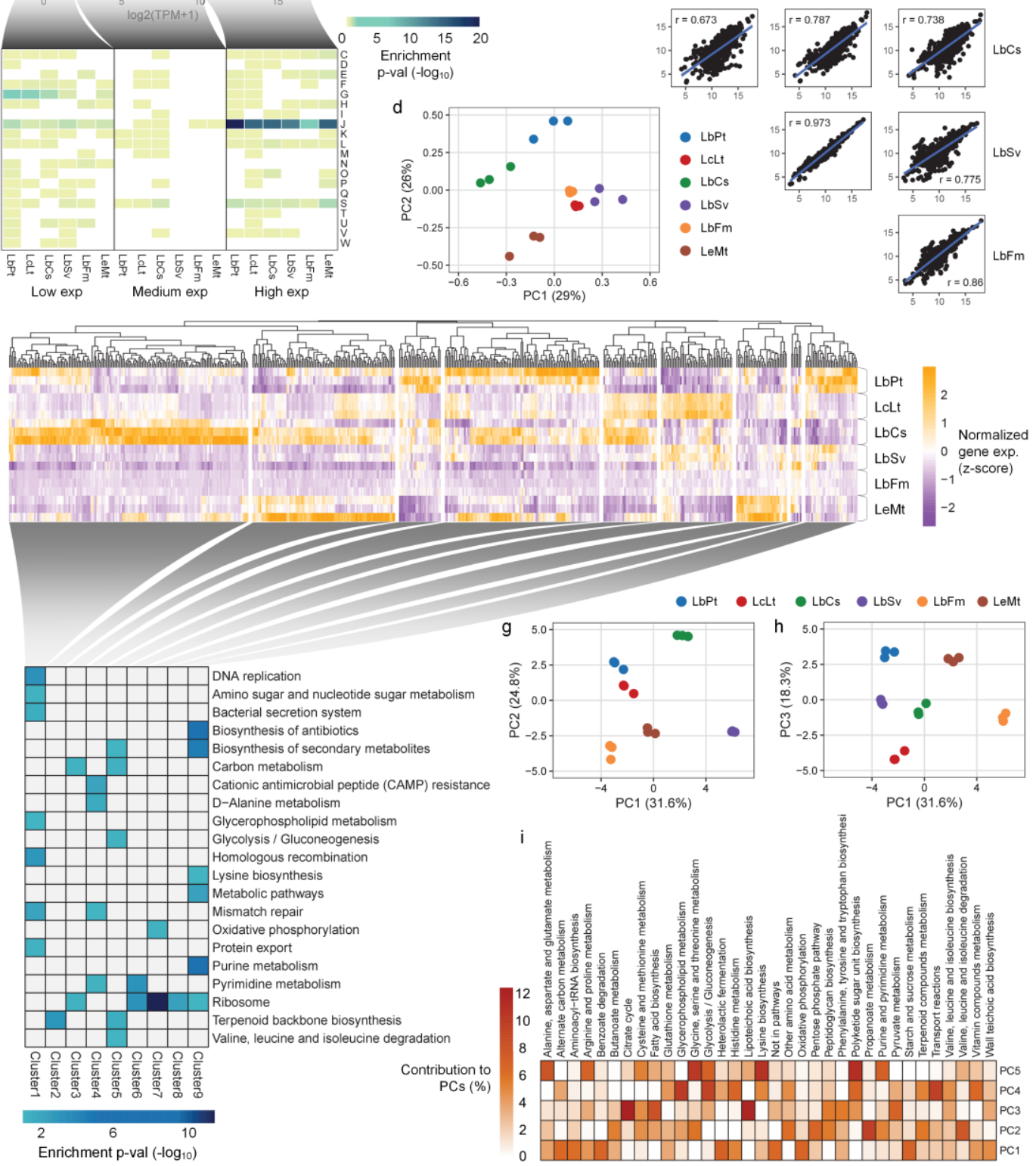

Figure 2. Transcriptomic characteristics of exponentially growing LAB in LABDM. (a) Cumulative distribution of gene expression values, (b) enrichment of NOG categories in three distinct gene expression ranges, i.e. low, medium and high, (c) plots showing pairwise correlation of gene expression of orthologous genes across various LAB, (d) principal component analysis (PCA) of the orthologous gene expression, (e) Hierarchical clustering of differentially expressed orthologous genes, (f) enrichment of KEGG pathways in differentially expressed genes and $(g-j) P C A$ of metabolic genes in various LAB strains. Numbers in (c) represent the Spearman correlation coefficient, $r$. The NOG term abbreviations in (b) are same as Figure 1. Negative $\log _{10}$ transformations of enrichment $p$-value is provided in (b) and (f). Clustering was done with Euclidean distances of normalized gene expressions (Z-scores) across LAB in (e). PCA of metabolic genes was performed based gene assignments to various pathways in newly reconstructed GEMs. 
a

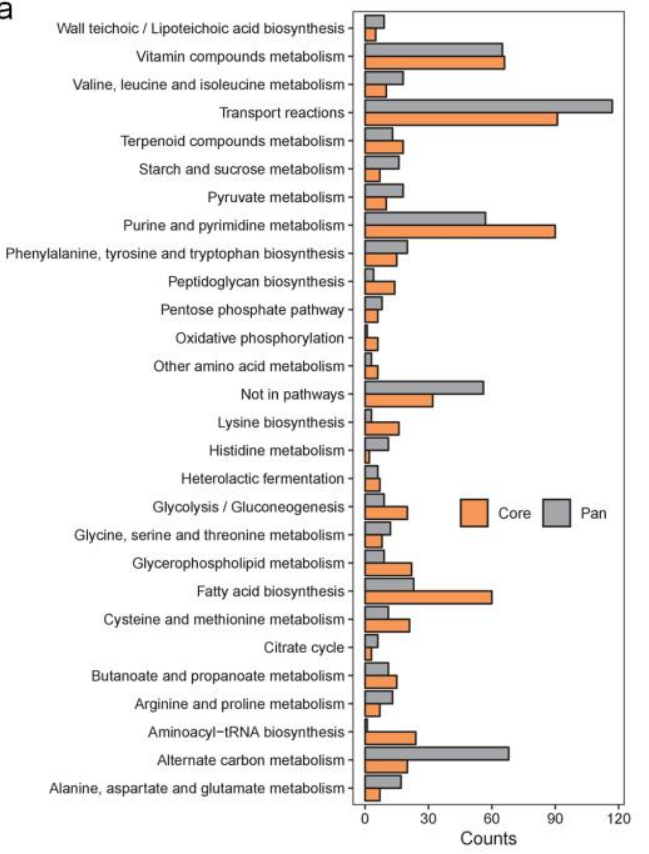

b

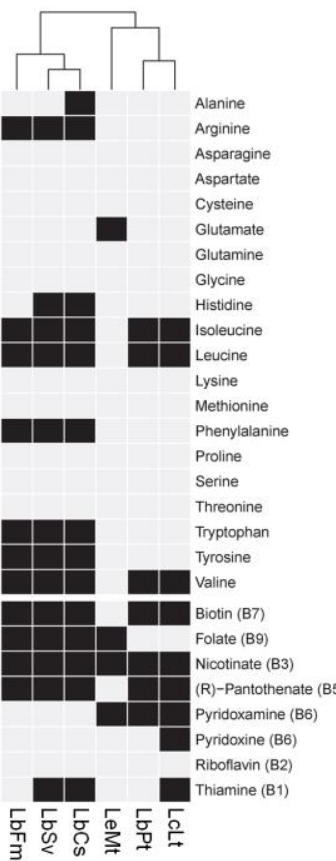

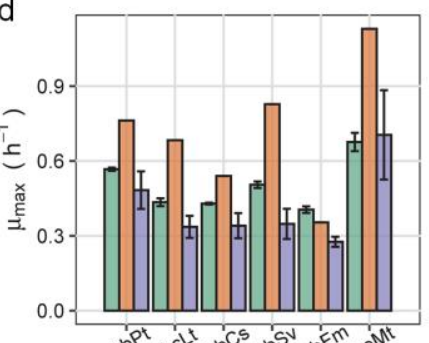

e

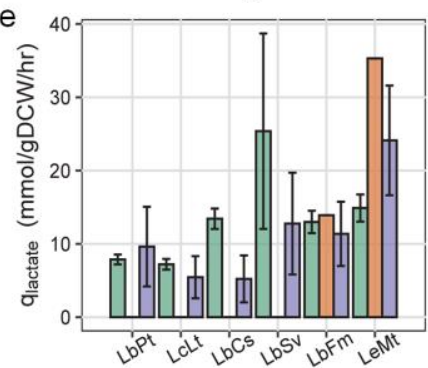

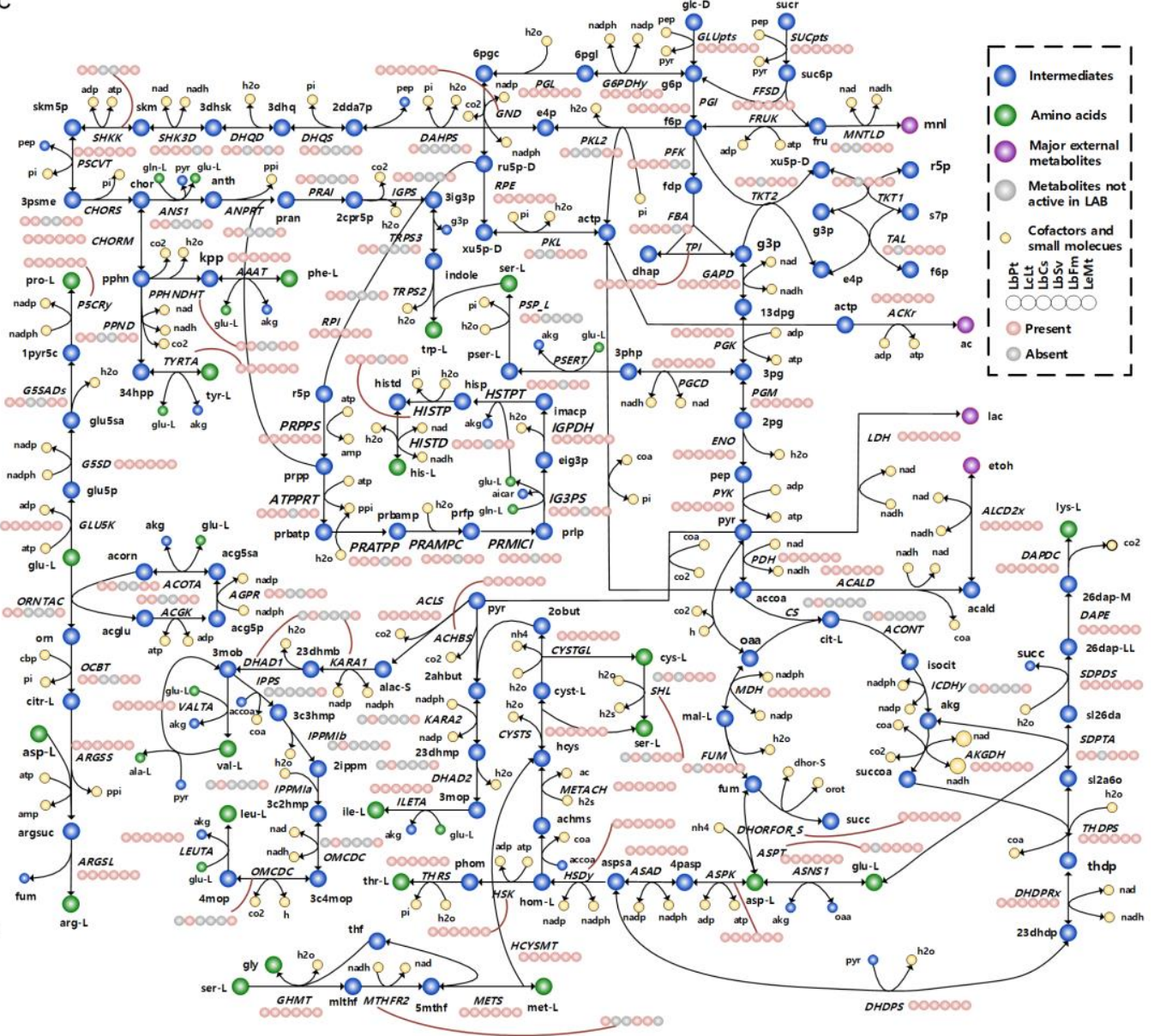

Figure 3. GEM characterized core and pan metabolic capabilities of LAB. (a) Distribution of core and pan reactions in different subsystems, (b) amino acid auxotrophy as revealed by LAB GEMs, (c) metabolic network showing the presence and absence of different reactions in central carbon metabolism and the amino acid biosynthesis pathways, (d) comparison of predicted and measured growth rates, (e) comparison of predicted and measured lactate production rates. The error bars in (d) and (e) denotes the standard deviation of triplicate measurements and the variations in predicted rates over multiple $a_{i}$ parameters in 5000 samples, respectively. 
bioRxiv preprint doi: https://doi org/10.1101/2021.06 20.449192 this version posted June 21, 2021. The copyright holder for this preprint (which was not certified by peer review) is the author/funder, who has granted bioRxiv a license to display the preprint in perpetuity. It is made available under aCC-BY-NC-ND 4.0 International license.

a
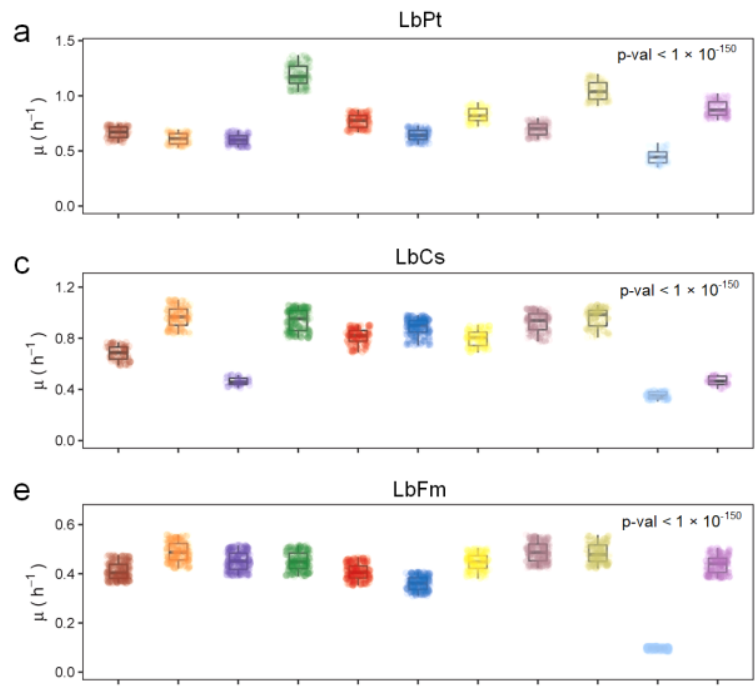

g b
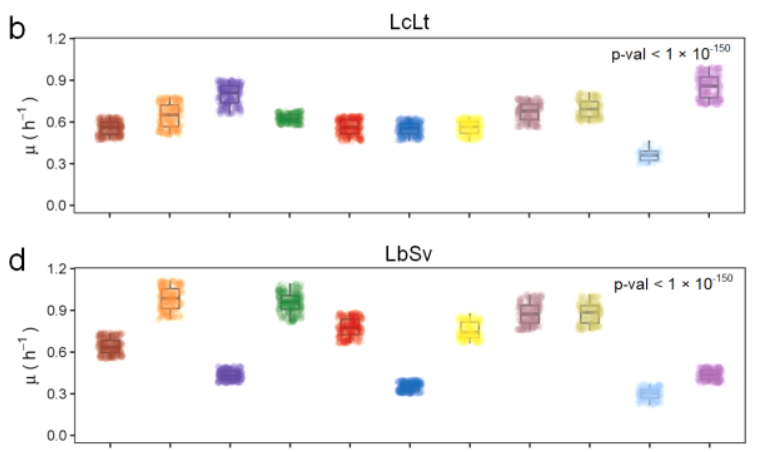

$\mathrm{f}$

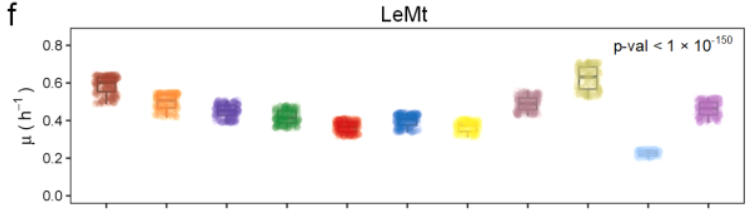

Normalized score

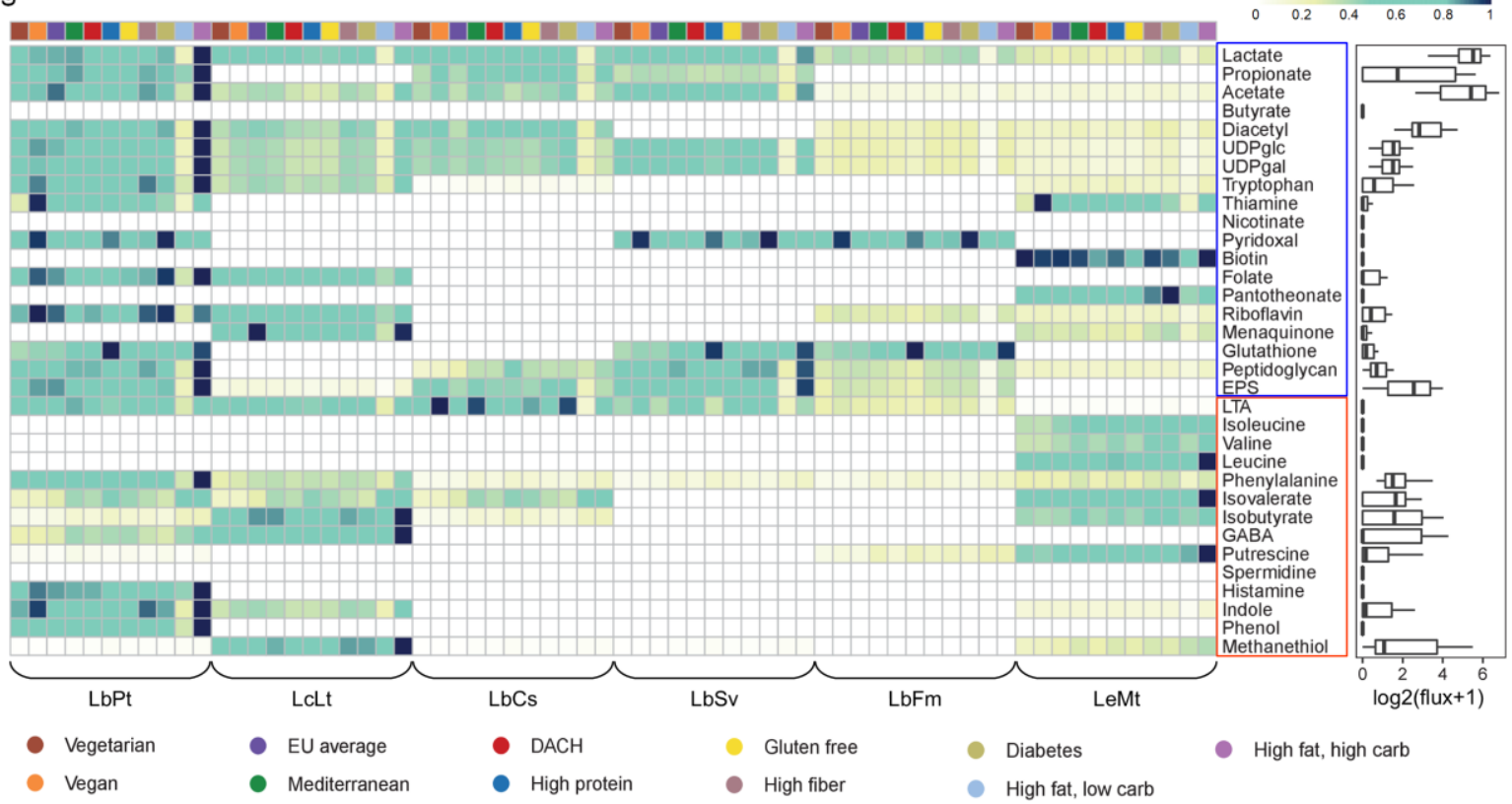

Figure 4. Diet-dependent probiotic characteristics of LAB simulated in silico. (a-f) Variations in simulated growth rates across various diets in each LAB species ( $p$-val $<1 \times 10^{-150}$, Kruskal-Wallis test), (g) ratio of positive to negative epistasis and $(\mathrm{h})$ postbiotic production capabilities of LAB in various diets. Growth rates in (a-f) is simulated by constraining each LAB GEM with respective diet conditions over 5000 samples. In $(\mathrm{g})$, the values in heatmap are production potential of each compound normalized to the maximum value across all diets and species. The boxplot in ( $\mathrm{g}$ ) shows the overall variation in production levels across all diets and species. Compounds colored in blue and red denote the beneficial and detrimental postbiotics, respectively. 
a

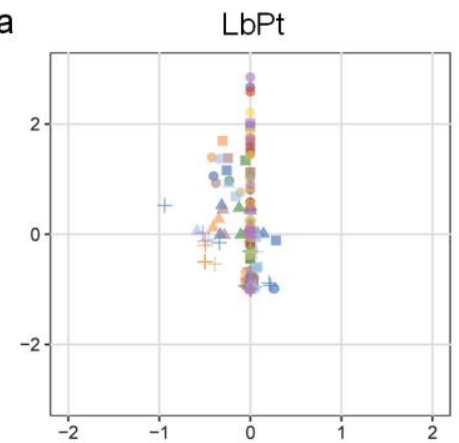

d

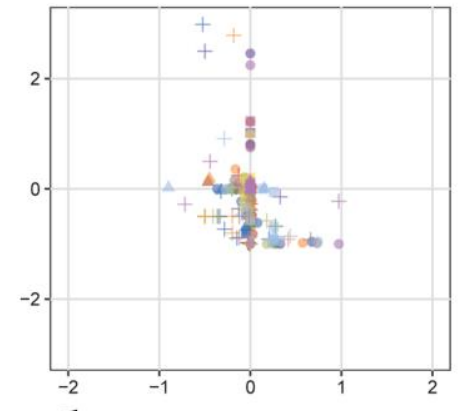

b

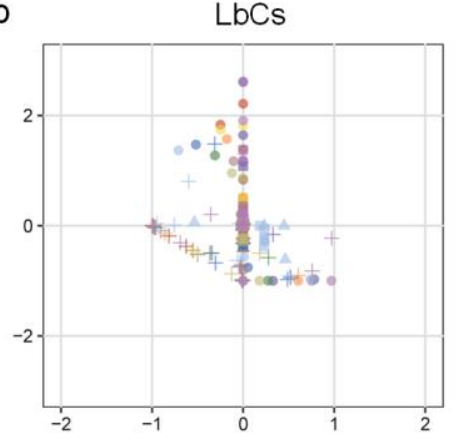

e

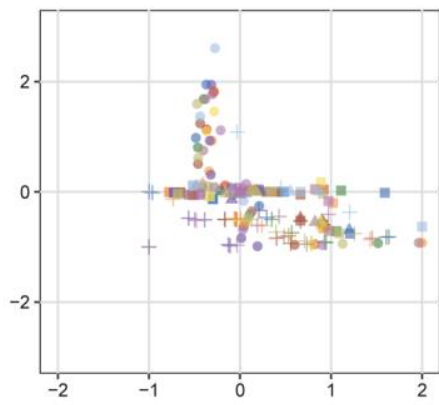

c

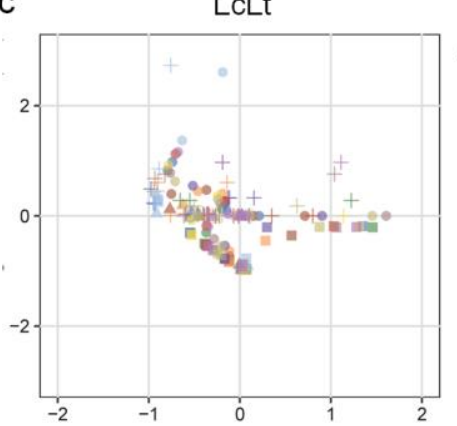

f

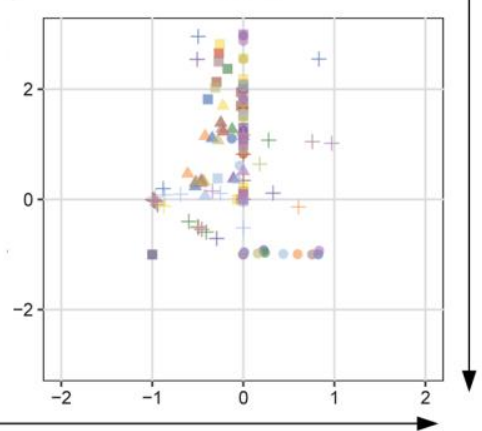

Change in growth rate of $L A B\left(\mathrm{~h}^{-1}\right)$

Vegetarian

- Vegan

- EU average

- Mediterranean
- DACH

- High protein

- Gluten free

- High fiber
Diabetes

- High fat, low carb

- High fat, high carb
- Commensal

- Opp. Pathogen

- Pathogen

+ Probiotic

Figure 5. Interactions of LAB with other gut microbes simulated in silico. (h-m) outcomes of pairwise metabolic interactions with various other gut microbes. The relative difference in the growth rate of each strain when it grows separately or in combination with another organism is plotted. The interactions of each $\angle A B$ was evaluated with 5 others and the following representative species: Probiotic - B. adolescentis; Commensal - A. muciniphila, B. thetaiotaomicron, E. faecalis, E. coli W3110, F. prausnitzii; Opp. Pathogen - P. putida, H. pylori, K. pneumoniae, P. aeruginosa, E. coli O157:H7; Pathogen $-S$. enterica ser. typhimurium, S. flexneri, S. sonnei, S. aureus. 
a

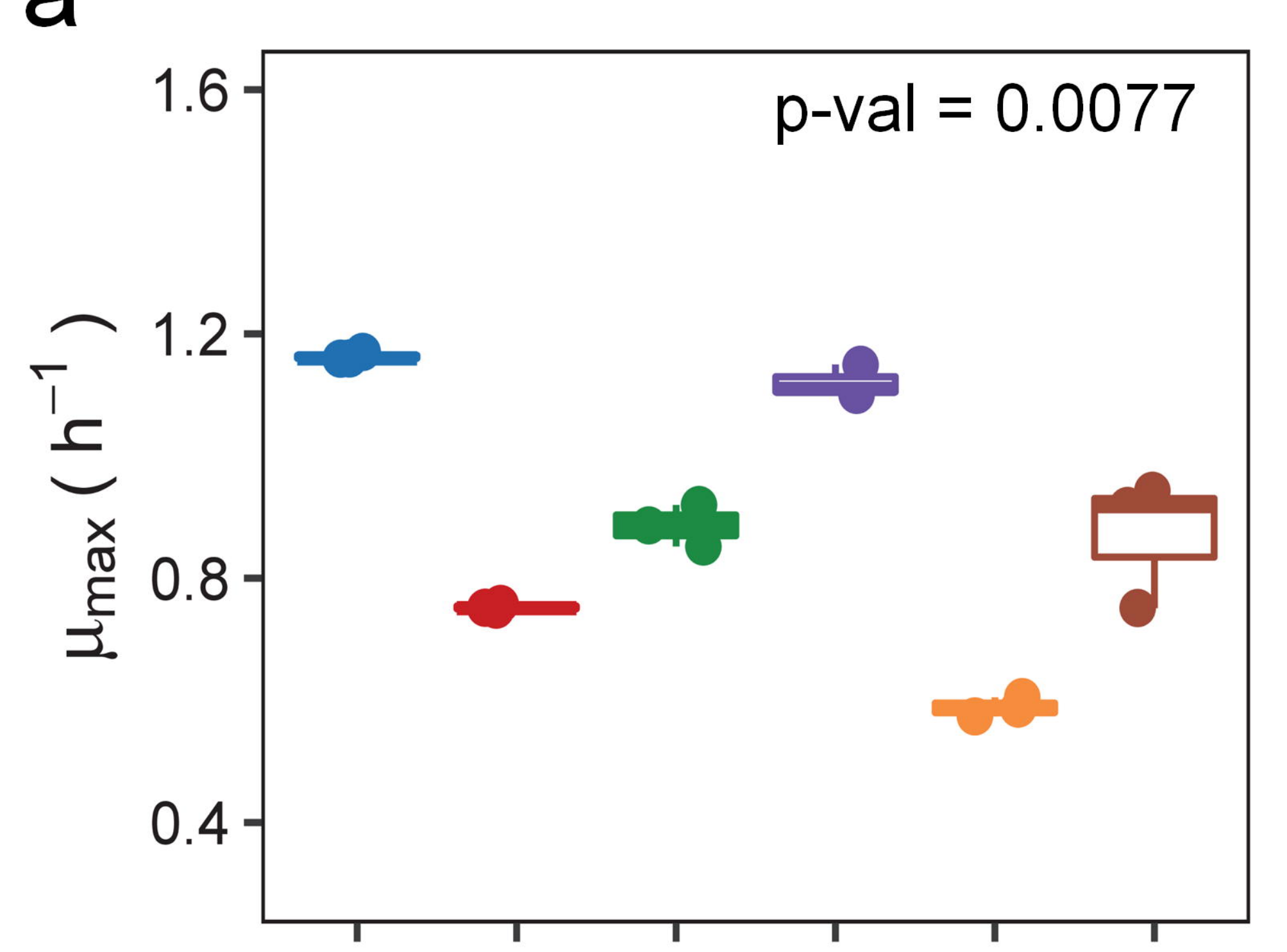

b

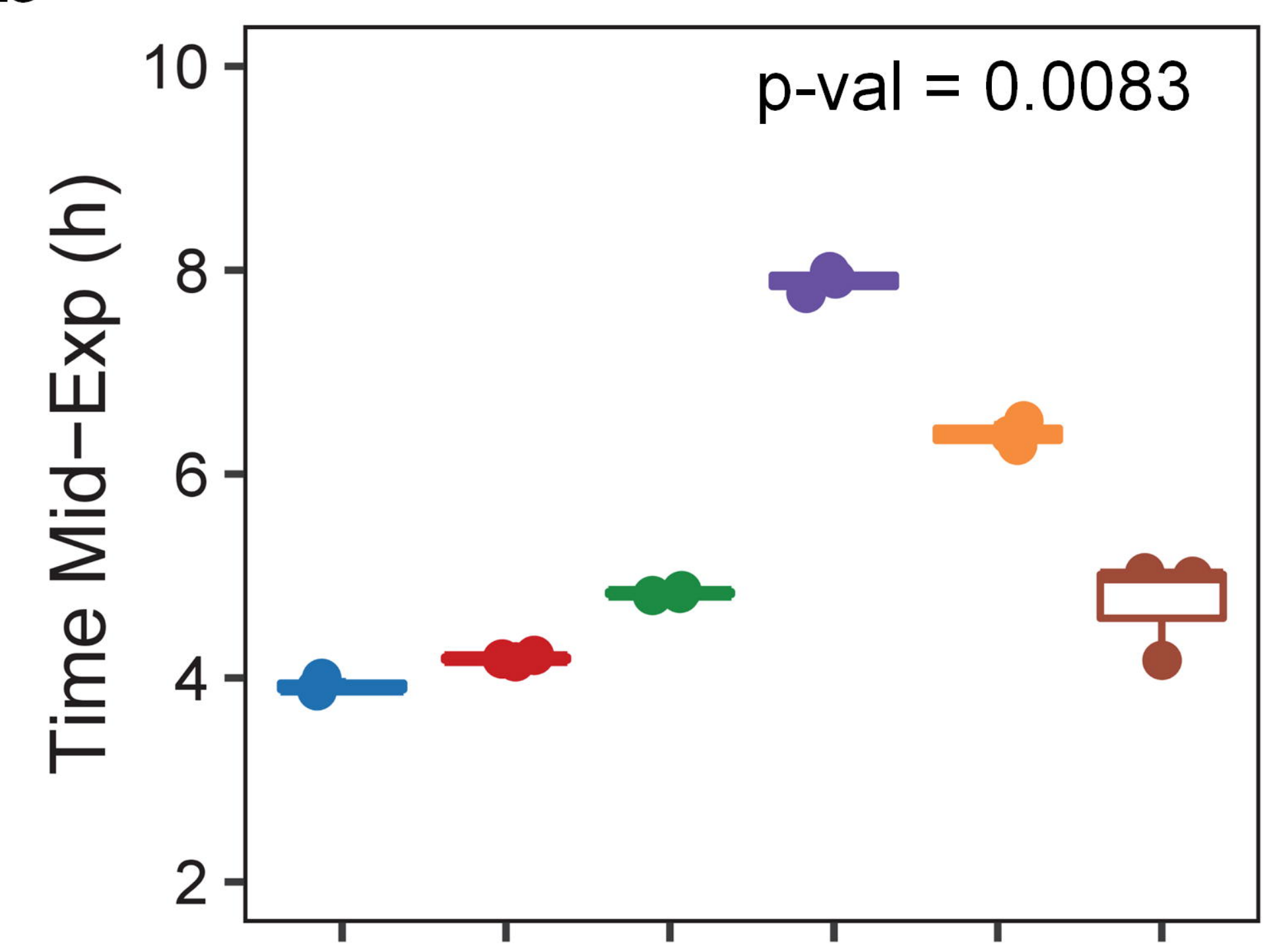

C

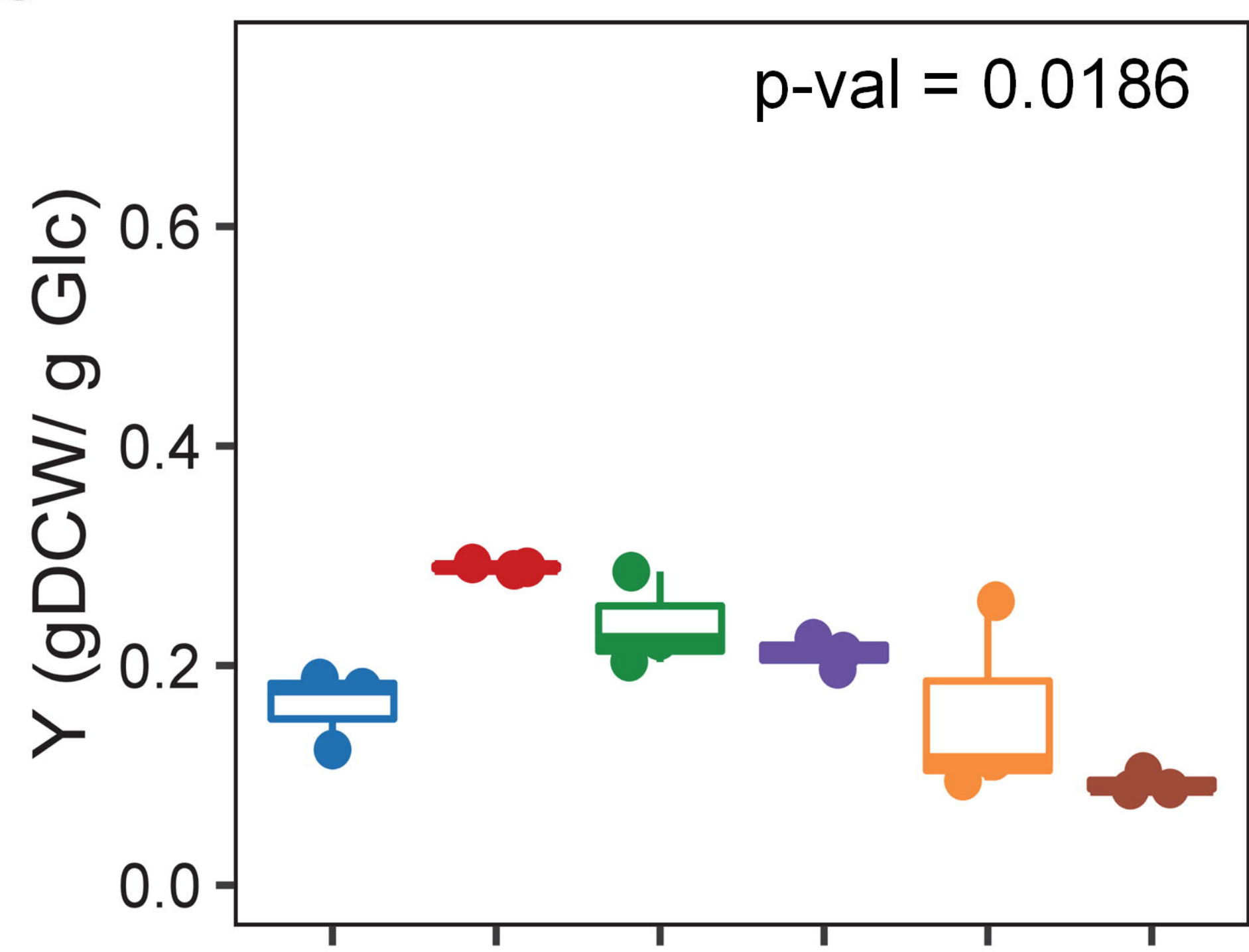

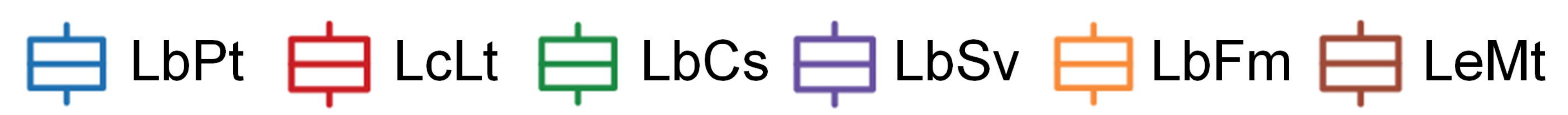

d

f

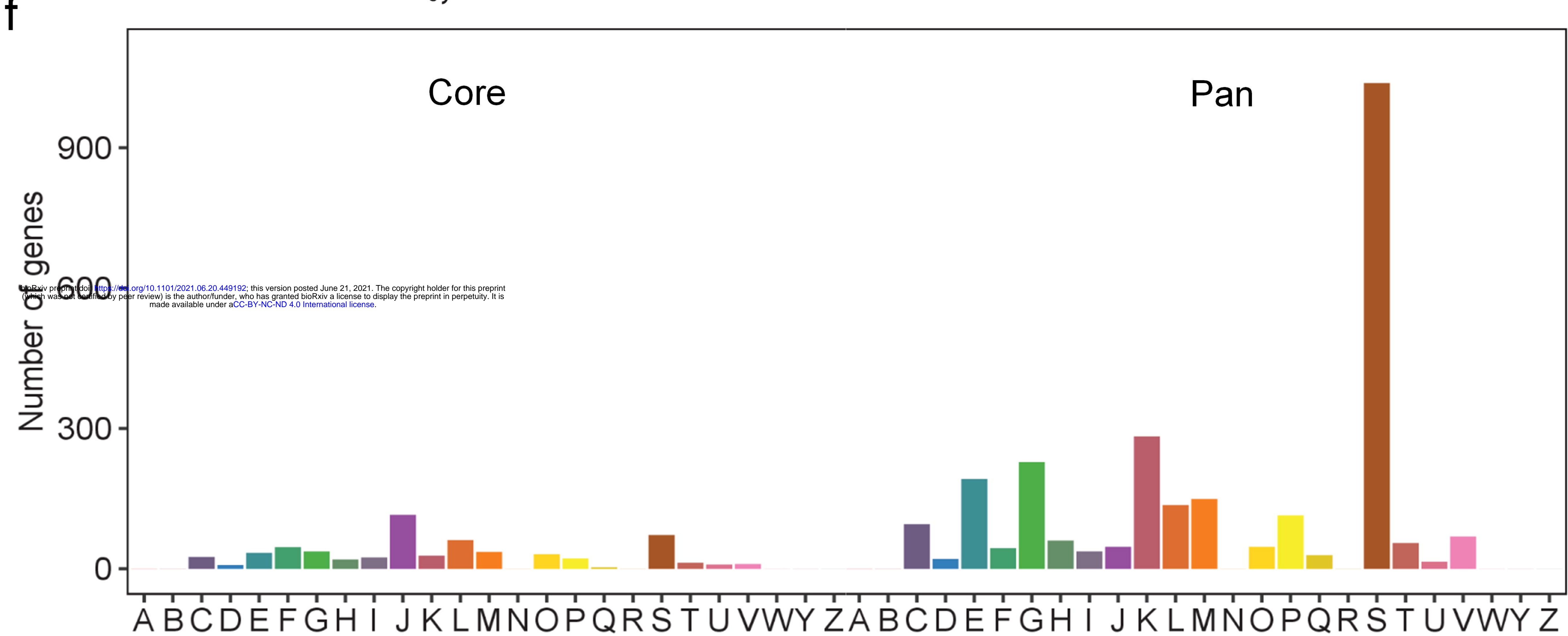

e

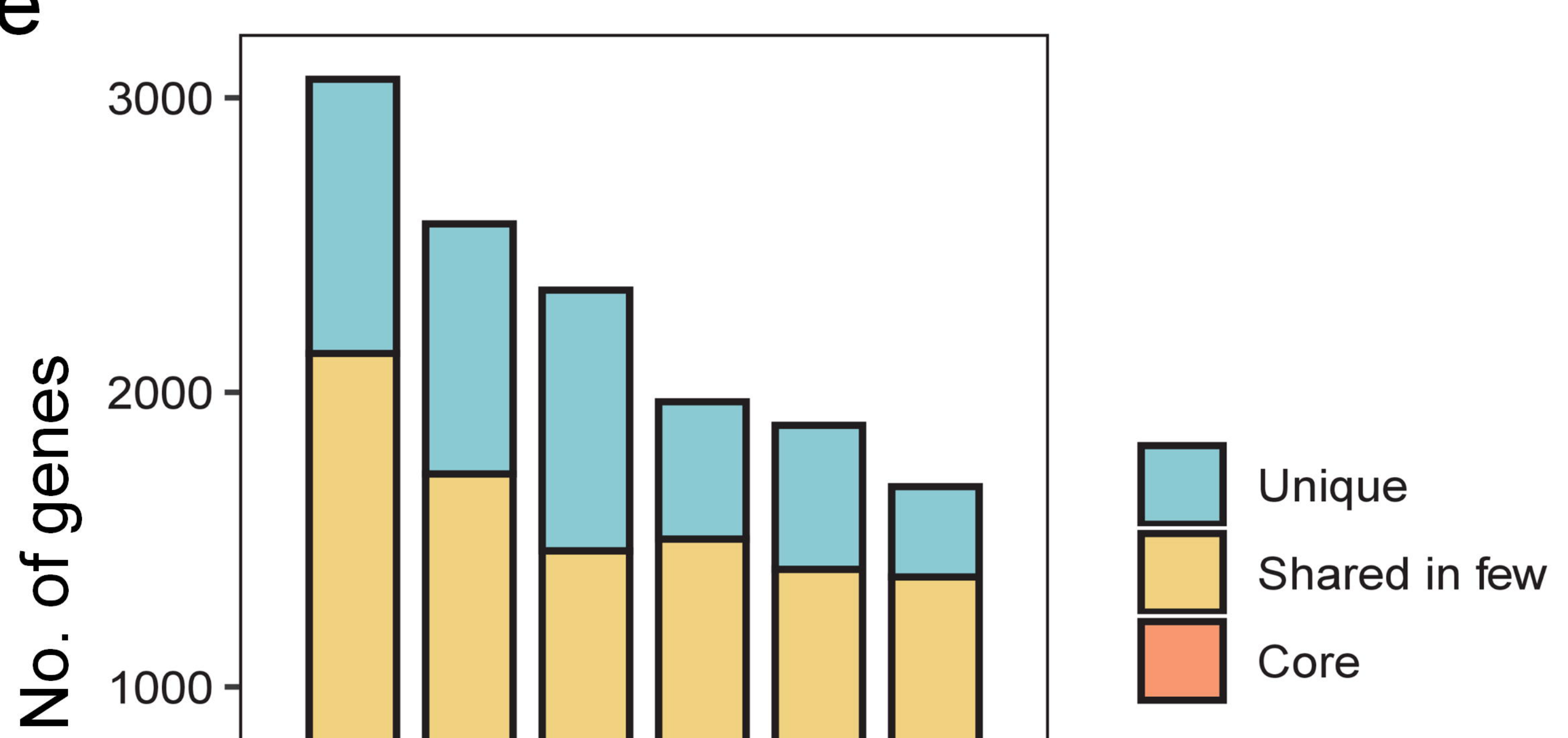

Tree scale: 0.1

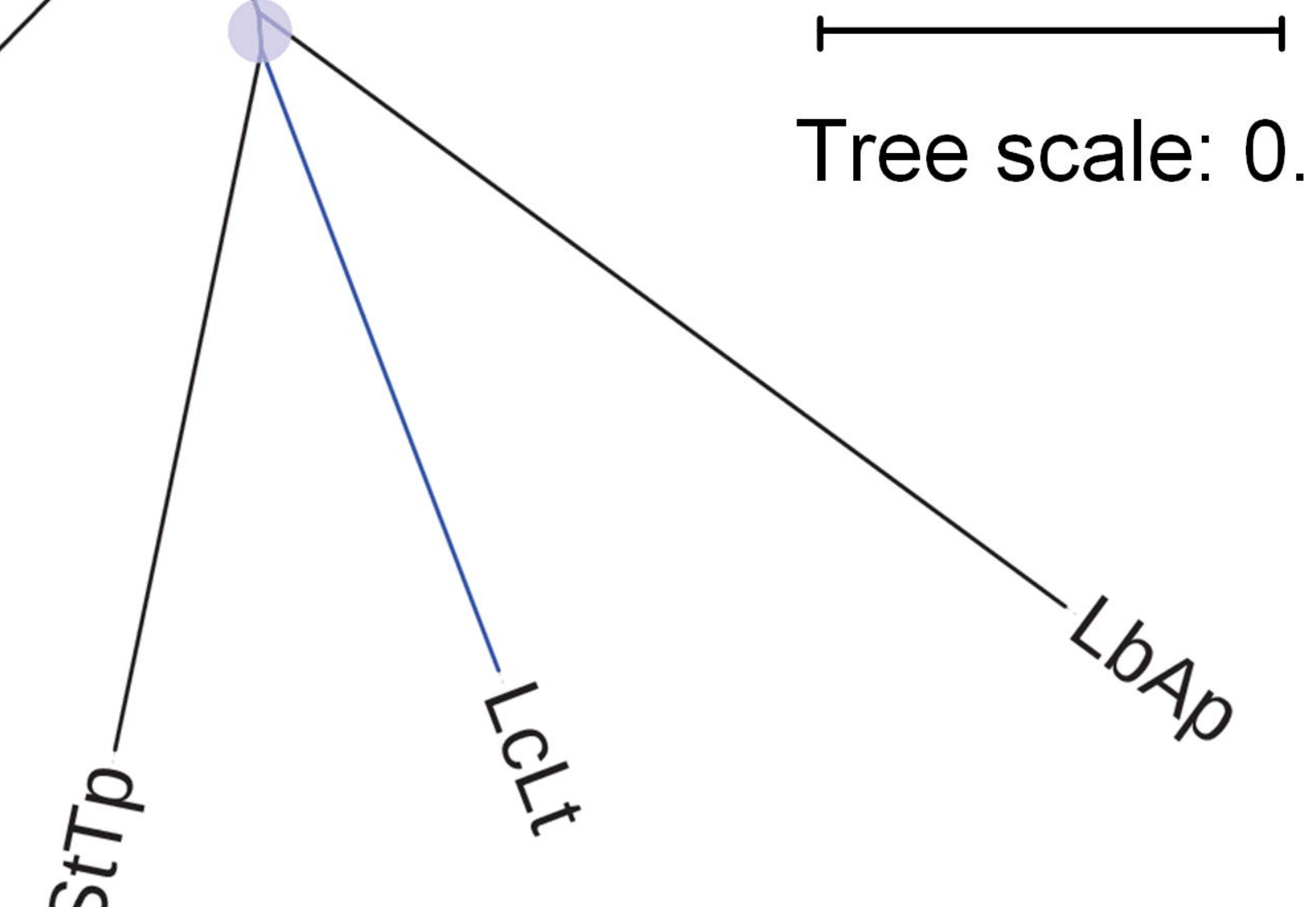

$0-$

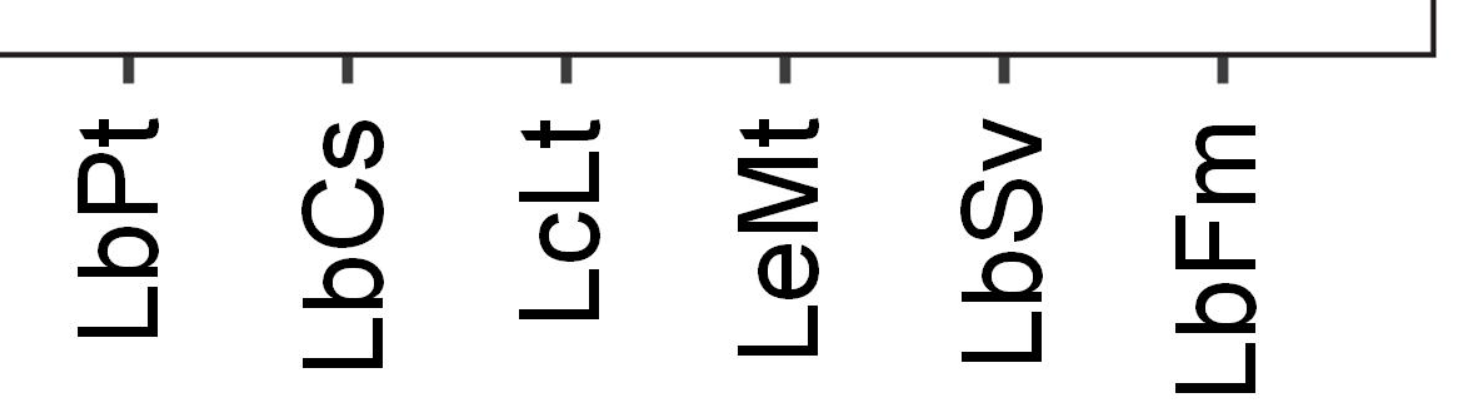

COG category

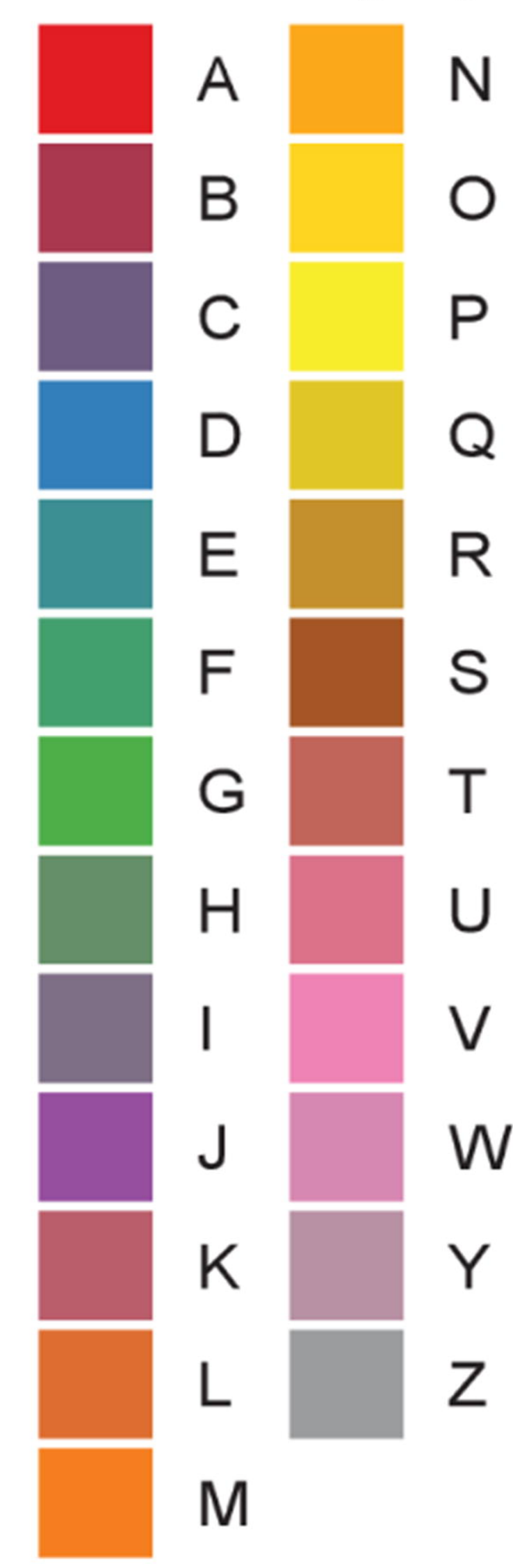

g
Bile resistance mechanisms $\quad 14$

Mucosal layer adhesion factors 12

Acid resistance systems 10

Protease systems (CEPs \& peptidases)

Resistance mehanisms to oxidative stress

Bacteriocins

LbFm LeMt 
a

a

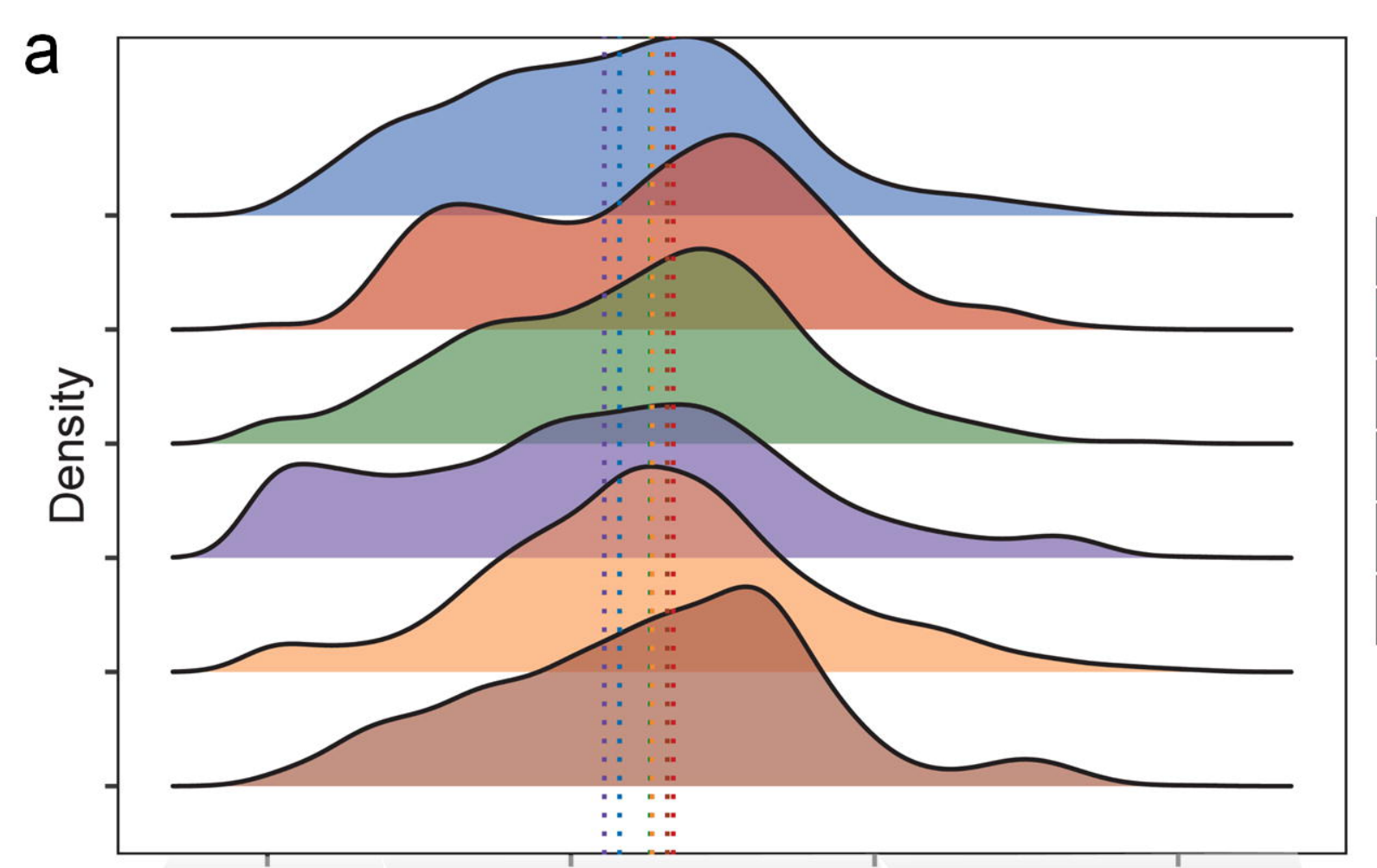

\section{b}

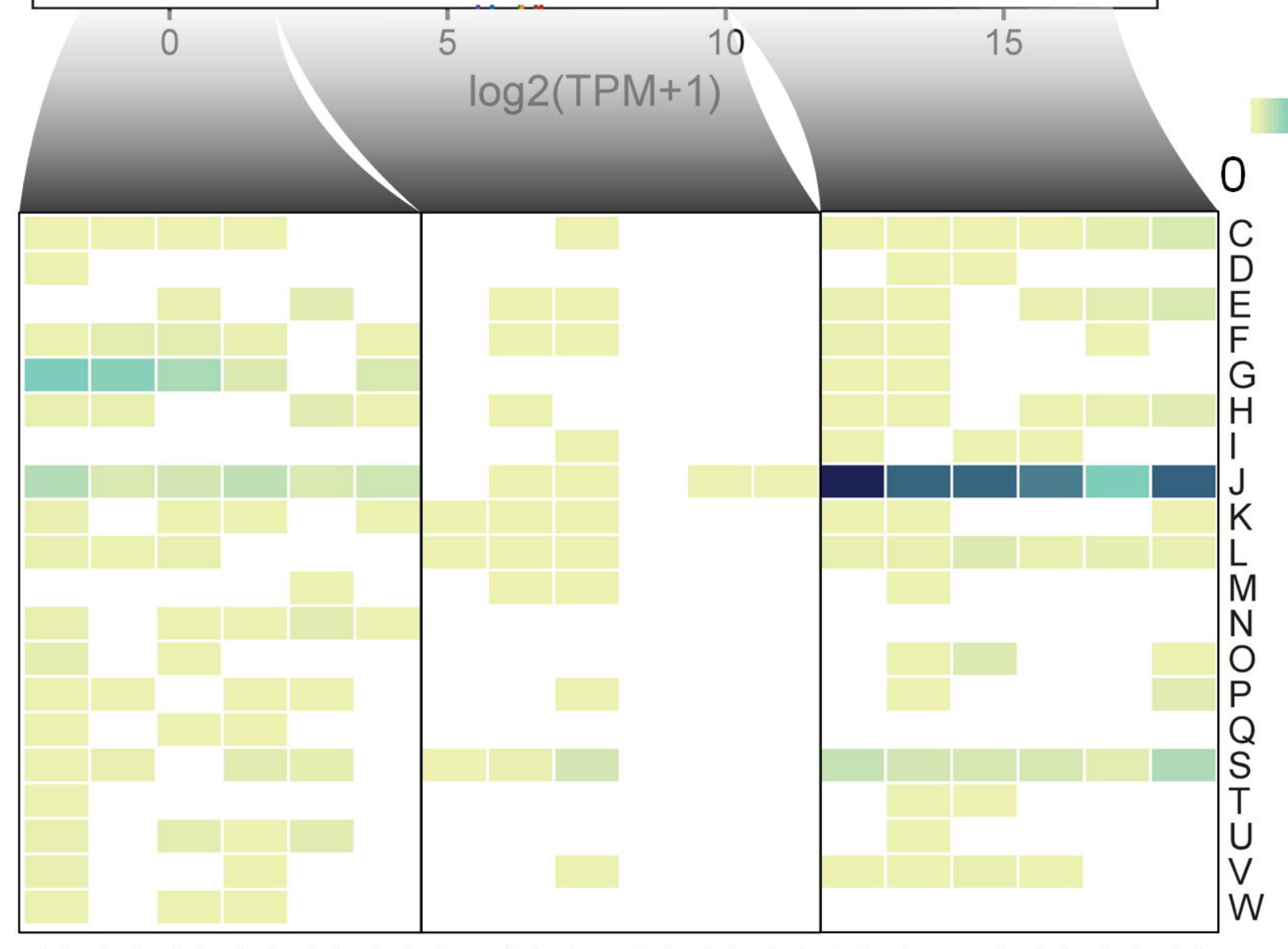

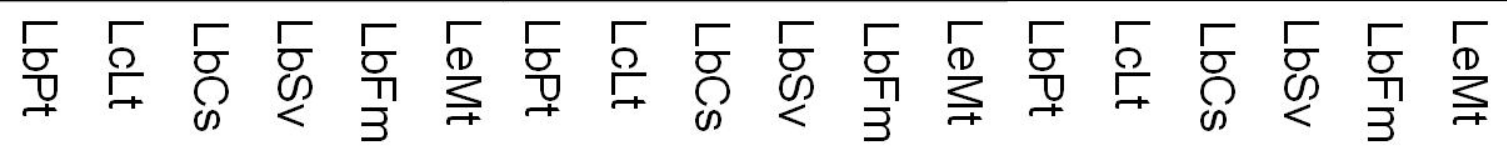
Low exp
Medium exp

High exp
C
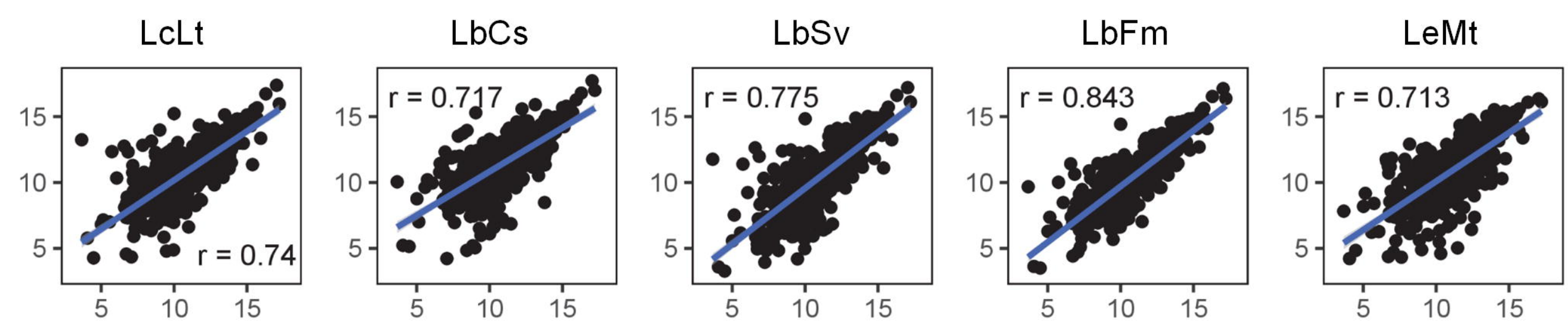

$\mathrm{LbPt}$
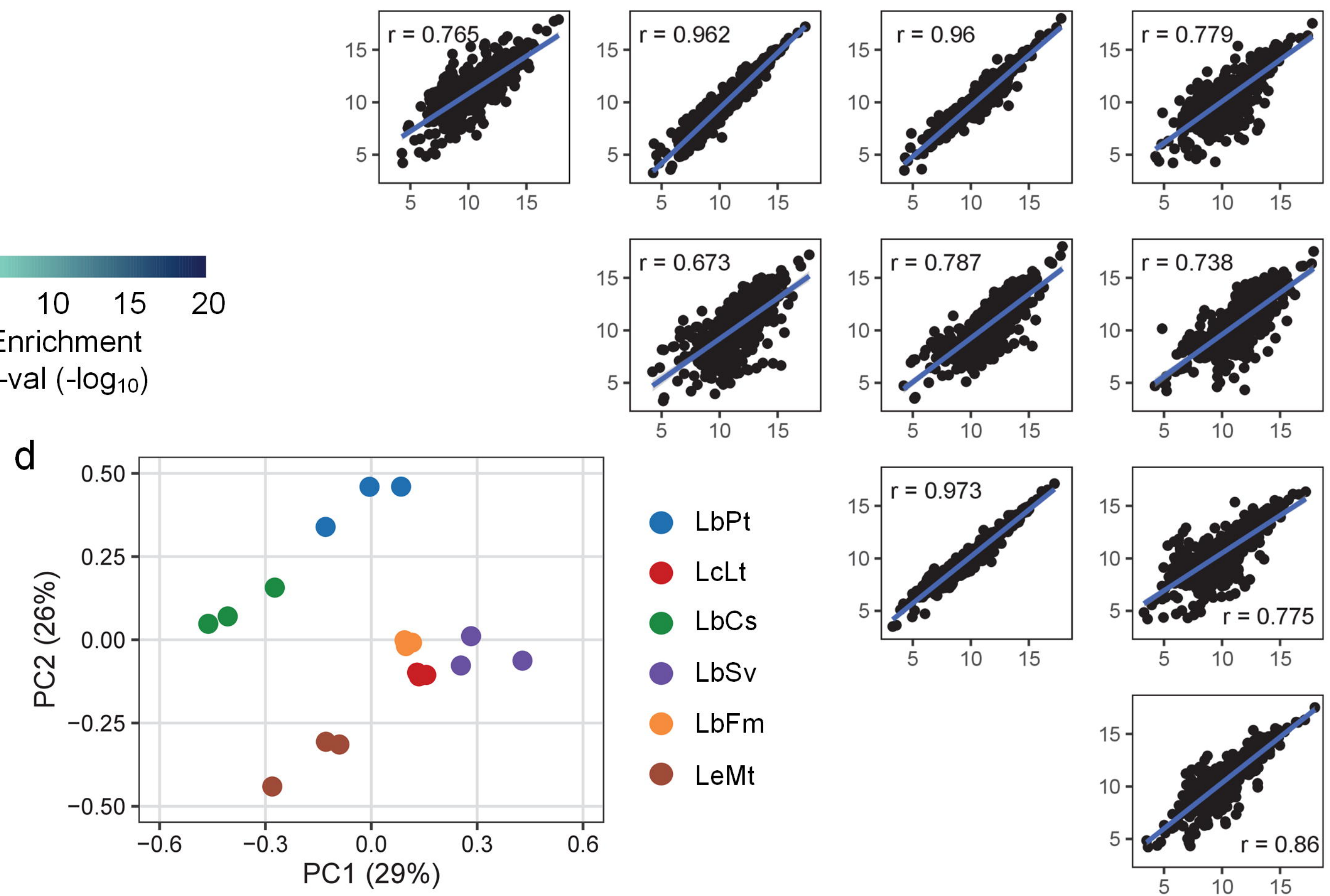

LbCs

\section{$\begin{array}{llll}5 & 10 & 15 & 20\end{array}$ \\ Enrichment \\ p-val $\left(-\log _{10}\right)$}

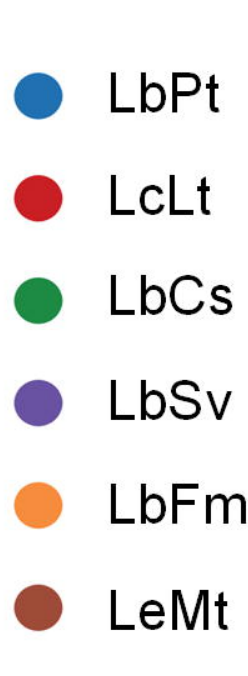

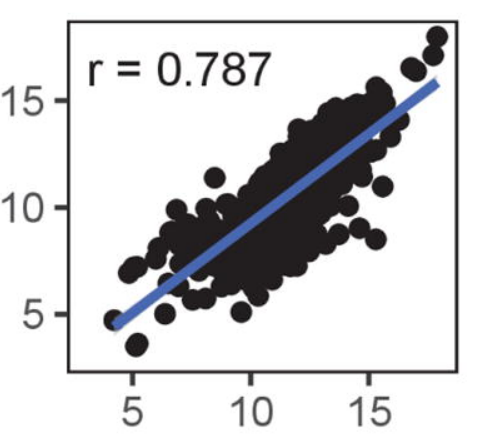
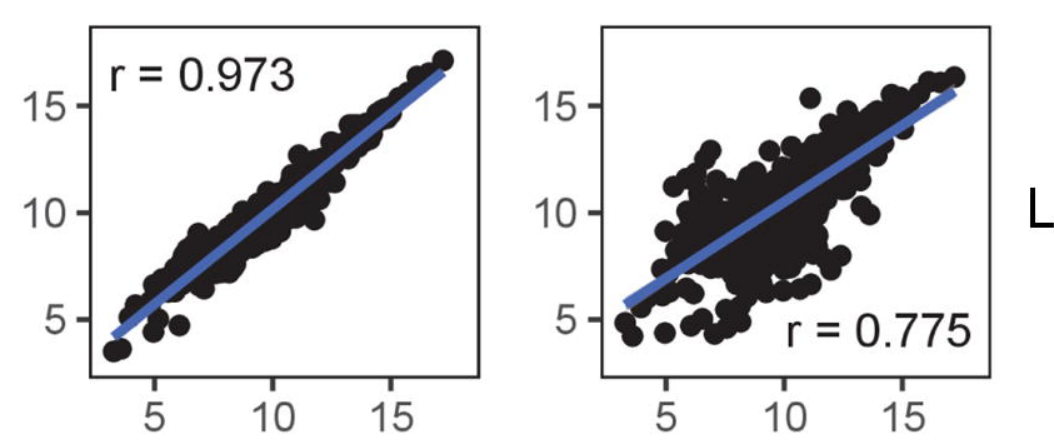

LbSv

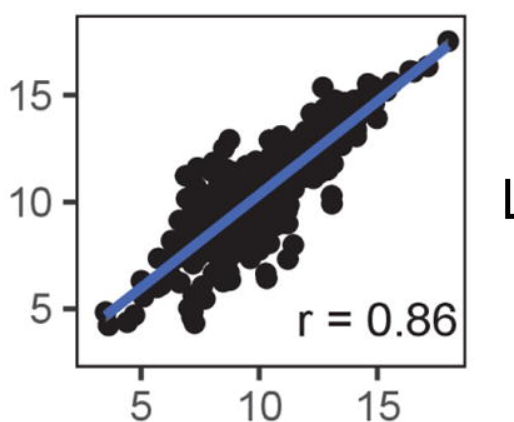

$\mathrm{LbFm}$

e

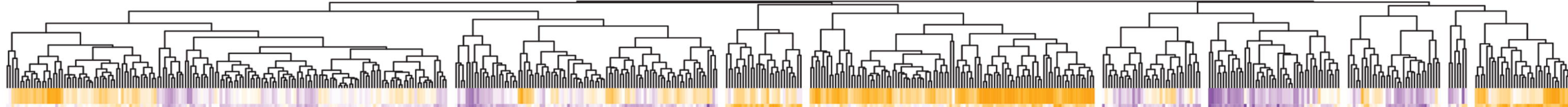
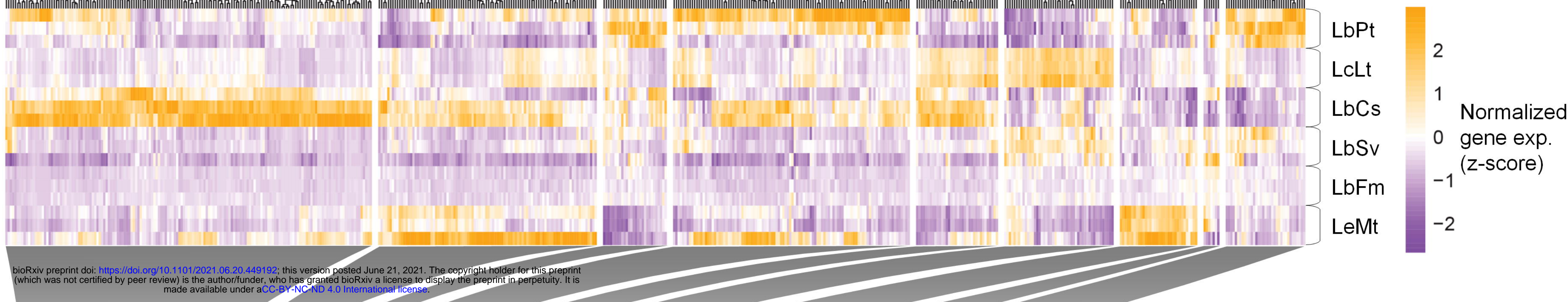

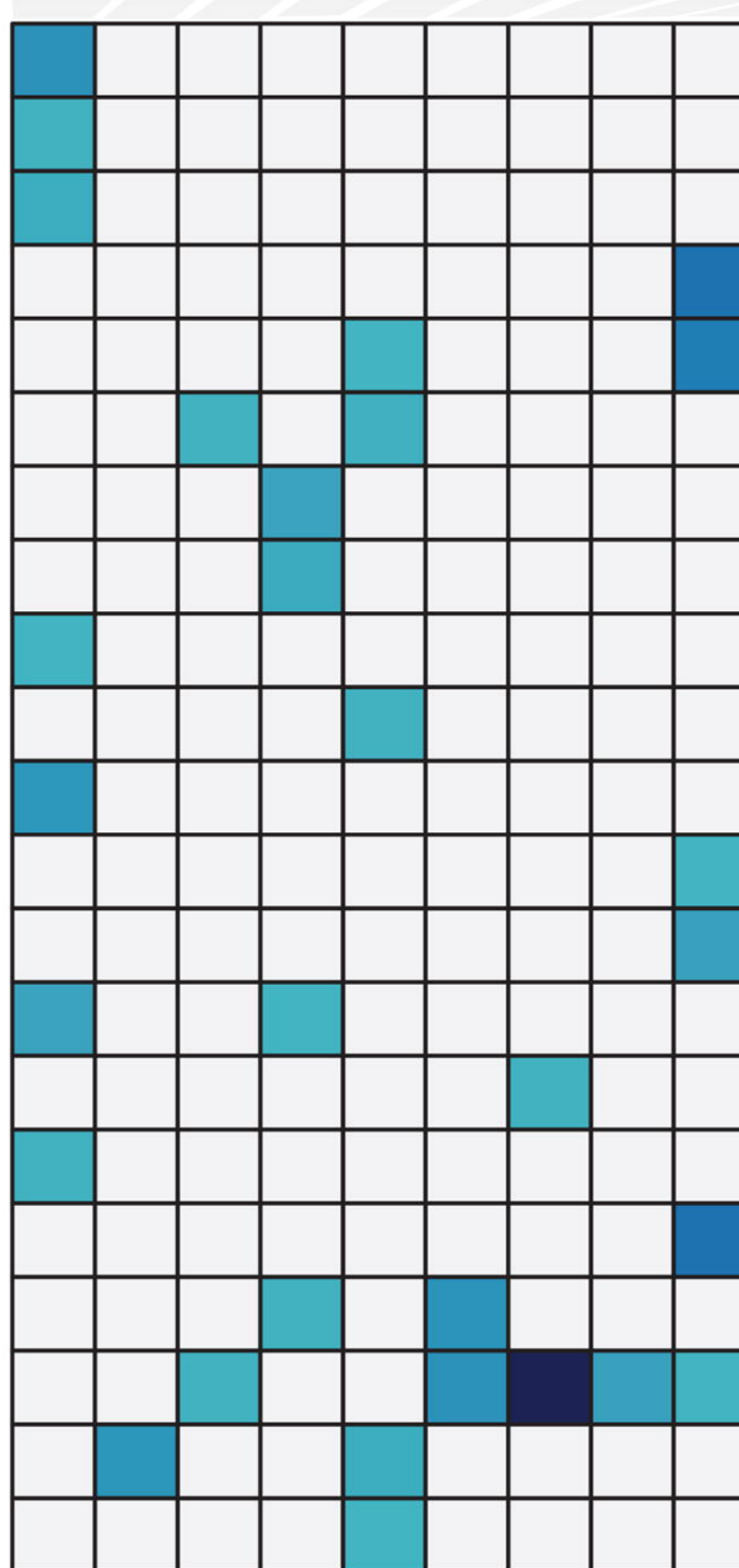

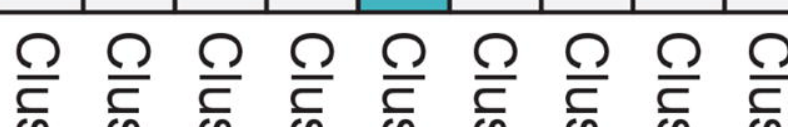

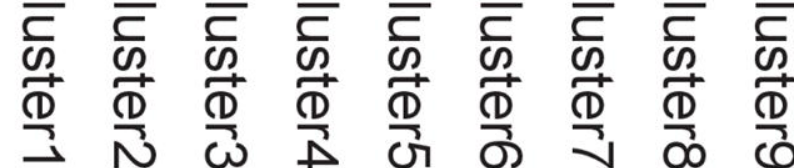

DNA replication

Amino sugar and nucleotide sugar metabolism Bacterial secretion system Biosynthesis of antibiotics Biosynthesis of secondary metabolites Carbon metabolism

Cationic antimicrobial peptide (CAMP) resistance D-Alanine metabolism

Glycerophospholipid metabolism Glycolysis / Gluconeogenesis Homologous recombination Lysine biosynthesis Metabolic pathways Mismatch repair Oxidative phosphorylation Protein export

Purine metabolism

Pyrimidine metabolism Ribosome

Terpenoid backbone biosynthesis

Valine, leucine and isoleucine degradation

Contribution to PCs (\%)
$\mathrm{LbPt}$
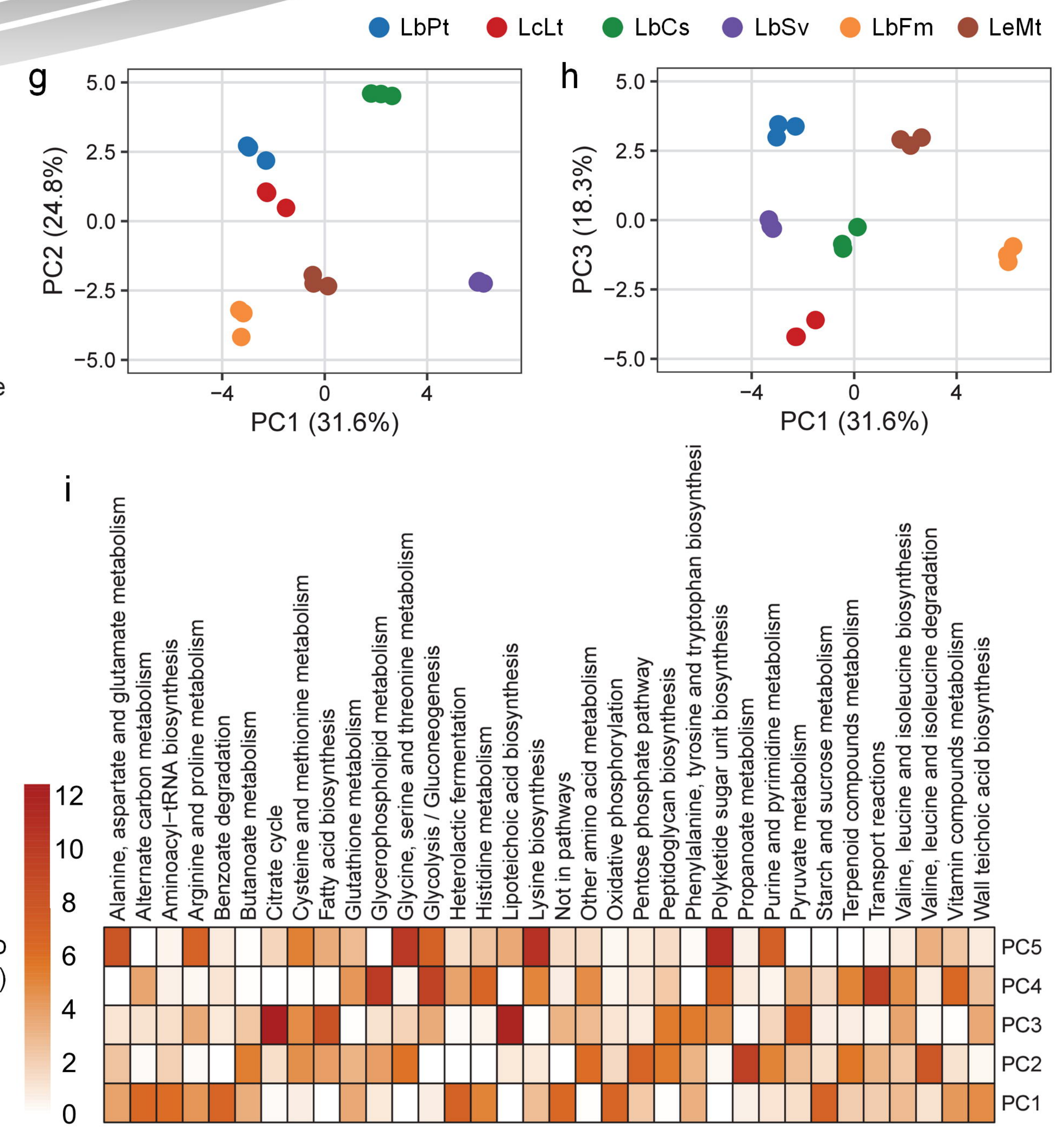$$
2
$$

$\begin{array}{lrr}2 & 6 & 10 \\ \text { Enrichment } p \text {-val }\left(-\log _{10}\right)\end{array}$ 
a

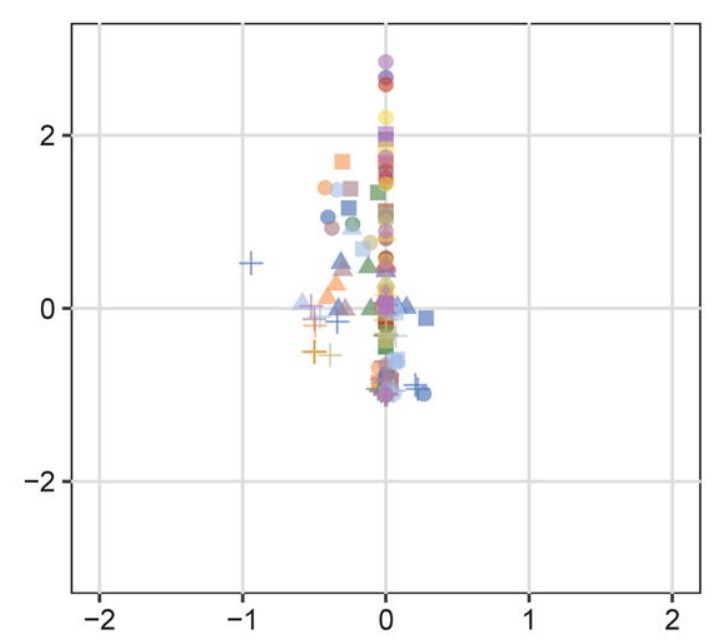

d

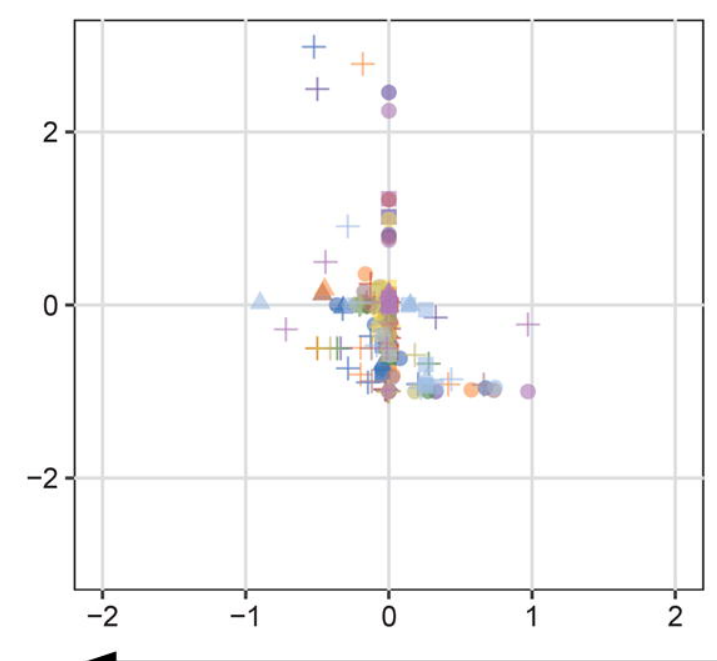

b

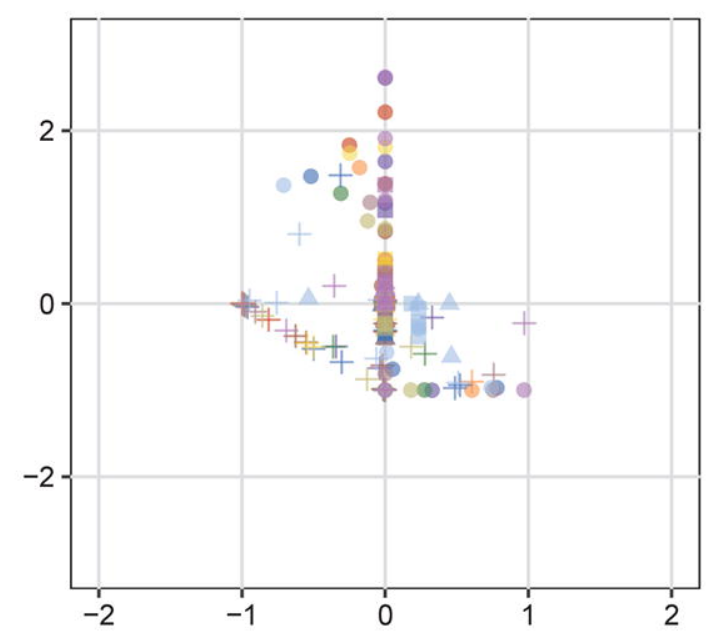

e

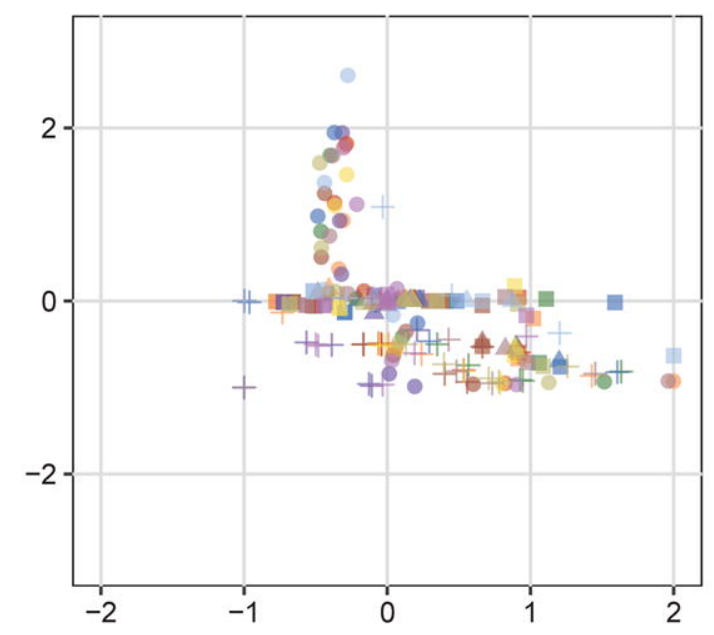

C

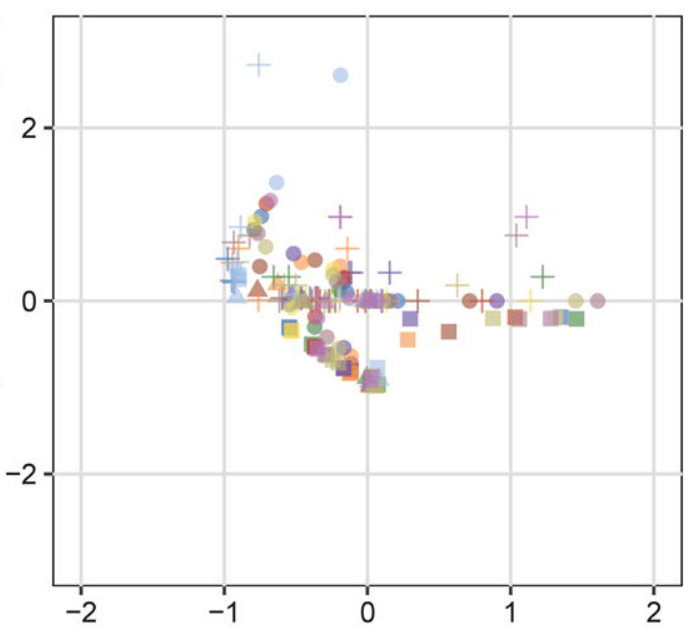

f

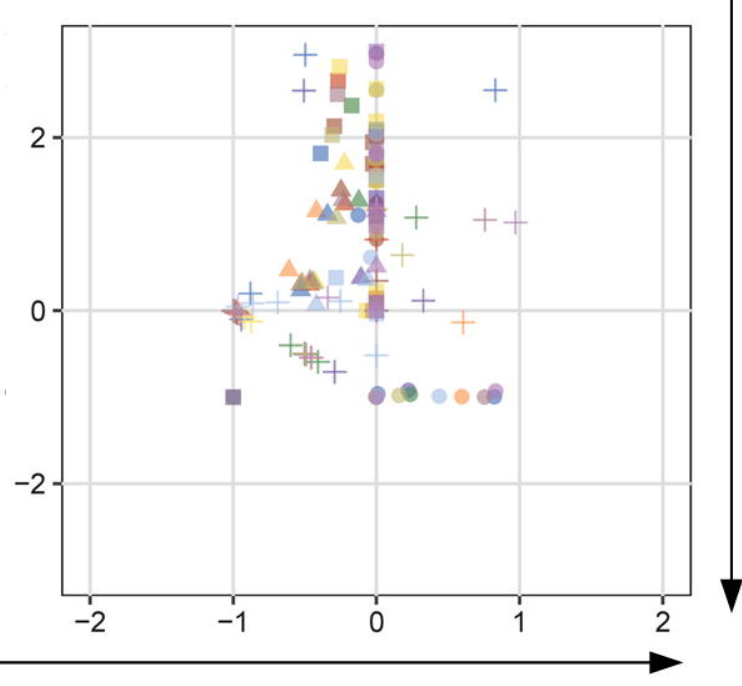

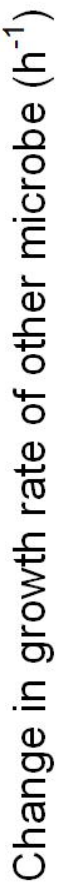

Change in growth rate of $\operatorname{LAB}\left(\mathrm{h}^{-1}\right)$

- Vegetarian

- Vegan

- EU average

- Mediterranean
- DACH

- High protein

- Gluten free

- High fiber
- Diabetes

- High fat, low carb

- High fat, high carb
- Commensal

- Opp. Pathogen

- Pathogen

+ Probiotic
Neutral Commensal

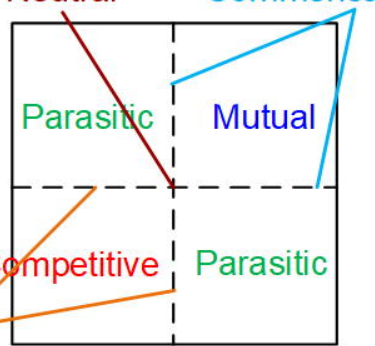


$b_{1}$

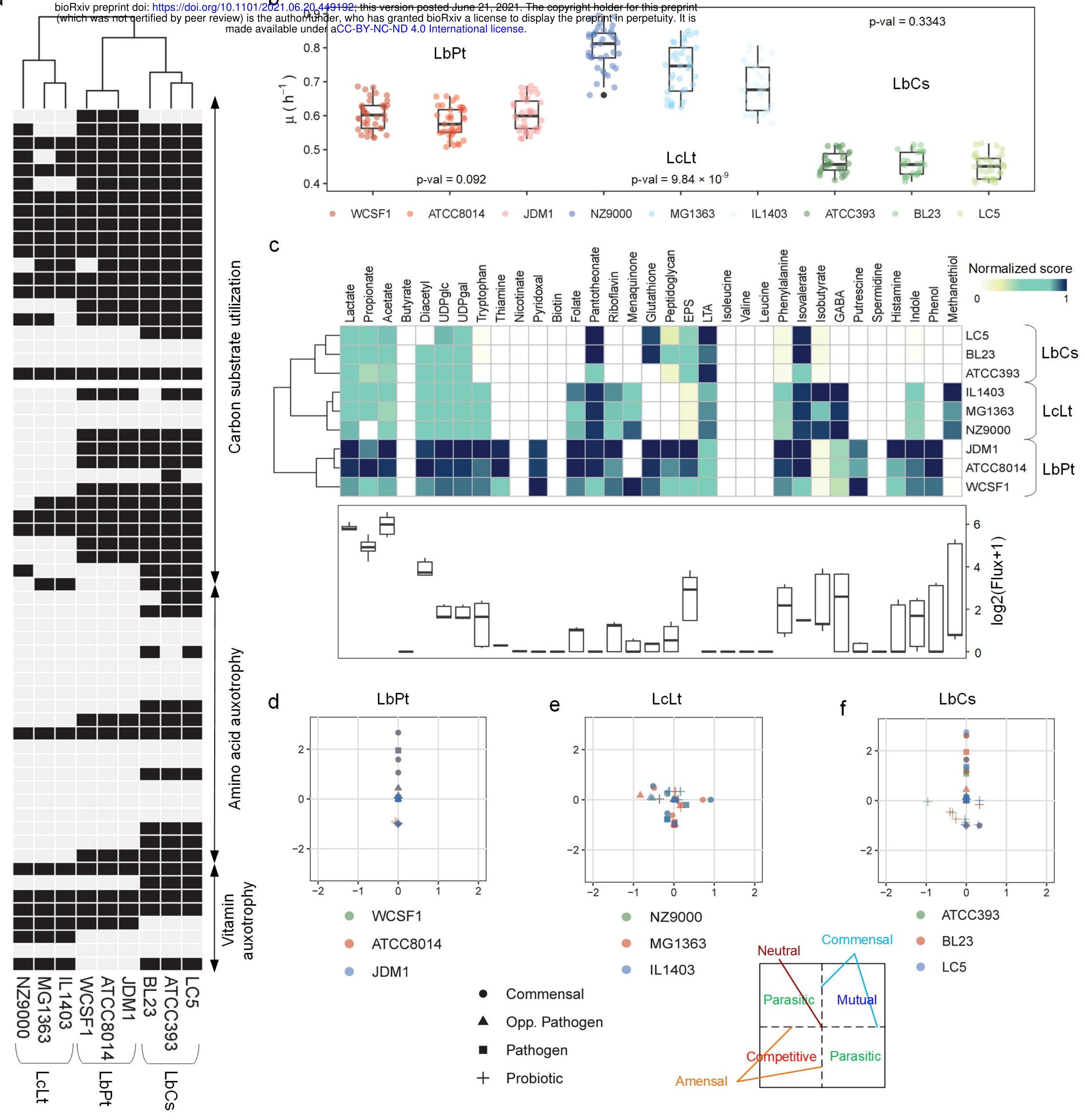

